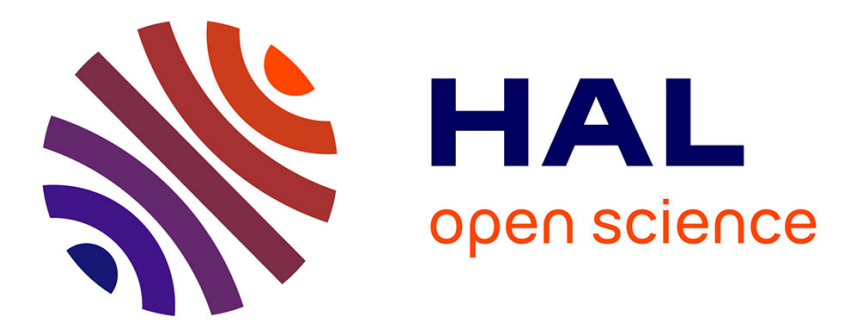

\title{
Multiobjective statistical learning optimization of RGB metalens
}

\author{
Mahmoud M R Elsawy, Anthony Gourdin, Mickael Binois, Régis Duvigneau, \\ Didier Felbacq, Samira Khadir, Patrice Genevet, Stéphane Lanteri
}

\section{- To cite this version:}

Mahmoud M R Elsawy, Anthony Gourdin, Mickael Binois, Régis Duvigneau, Didier Felbacq, et al.. Multiobjective statistical learning optimization of RGB metalens. ACS photonics, 2021, 8 (8), pp.24982508. 10.1021/acsphotonics.1c00753 . hal-03212349v2

HAL Id: hal-03212349

https://hal.science/hal-03212349v2

Submitted on 25 May 2021

HAL is a multi-disciplinary open access archive for the deposit and dissemination of scientific research documents, whether they are published or not. The documents may come from teaching and research institutions in France or abroad, or from public or private research centers.
L'archive ouverte pluridisciplinaire HAL, est destinée au dépôt et à la diffusion de documents scientifiques de niveau recherche, publiés ou non, émanant des établissements d'enseignement et de recherche français ou étrangers, des laboratoires publics ou privés. 


\title{
Multiobjective statistical learning optimization of RGB metalens
}

\author{
Mahmoud M. R. Elsawy, ${ }^{*, \dagger}$ Anthony Gourdin, ${ }^{\dagger}$ Mickaël Binois, ${ }^{\dagger}$ Régis

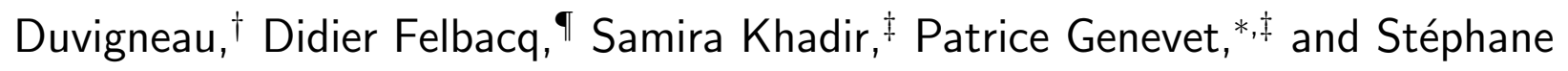 \\ Lanteri $^{\dagger}$ \\ †Université Côte d'Azur, Inria, CNRS, LJAD, 06902 Sophia Antipolis Cedex, France \\ $\ddagger C N R S$, CRHEA, Université Côte d'Azur, Sophia Antipolis, Valbonne, France \\ \Laboratoire Charles Coulomb, UMR5221 CNRS-Université de Montpellier, 34095 \\ Montpellier, France
}

E-mail: mahmoud.elsawy@inria.fr; patrice.genevet@crhea.fr

\begin{abstract}
Modelling of multi-wavelength metasurfaces relies on adjusting the phase of individual nanoresonators at several wavelengths. The traditional procedure neglects the near-field coupling between the nanoresonators, which dramatically reduces the overall diffraction efficiency, bandwidth, numerical aperture and device diameter.Another alternative design strategy is to combine a numerical optimization technique with fullwave simulations to mitigate this problem and optimize the entire metasurface at once. Here, we present a global multiobjective optimization technique that utilizes statistical learning method to optimize RGB spherical metalenses at the visible wavelengths. The optimization procedure, coupled to a high-order full-wave solver, accounts for the near field coupling between the resonators. High numerical aperture RGB lenses(NA=0.47 and $\mathrm{NA}=0.56$ ) of $8 \mu \mathrm{m}$ and $10 \mu \mathrm{m}$ diameters are optimized with numerical average
\end{abstract}


focusing efficiencies of $55 \%$ and $45 \%$, with an average focusing error smaller than $6 \%$ for the RGB colors. The fabricated and experimentally characterized devices present $44.16 \%$ and $31.5 \%$ respective efficiencies. The reported performances represent the highest focusing efficiencies for high $N A>0.5$ RGB metalenses obtained so far. The integration of multi-wavelength metasurfaces in portable and wearable electronic devices requires high performances to offer a variety of applications ranging from classical imaging to virtual and augmented reality.

\section{Introduction}

Metasurfaces, composed of arrangement of subwavelength resonators with different geometrical parameters, are attracting widespread interest notably due to their distinctiveness in controlling light properties in very short propagation distances.$^{1-8}$ Their integration in novel technologies led to exceptional and peculiar applications ${ }^{9}$ in relation to laser wavefront engineering,${ }^{10}$ sub-diffraction optical microscopy,${ }^{11}$ nonlinear optics, ${ }^{12}$ and quantum optics, ${ }^{13}$ to cite a few examples.

Precise engineering of subwavelength patterning is compulsory in enhancing the performance of metasurfaces. Several innovative inverse design methodologies have recently been exploited to address this issue, but only considering one metasurface functionality . $^{14-17}$ Today, we are witnessing a noteworthy demand in designing flat optics devices with a wide variety of responses to realize multifunctional metasurfaces. This makes the exploitation of rigorous inverse design strategies for multiple functionalities mandatory for the next metasurface generation.

In most cases, achieving the best fit in one objective or functionality degrades the performance of the other objectives. Multiobjective optimization capable of optimizing a problem with multiple competing objectives in a large parameter space is needed, but it is not a straightforward task. Two major approaches have been proposed to address this problem.

The first strategy essentially relies on transforming the multiobjective optimization prob- 
lem into a single objective one. This is realized by merging different objectives via a classical weighted sum technique. ${ }^{18-20}$ In most cases, the single objective problem has been tackled utilising classical evolutionary techniques. ${ }^{21-24}$ This straightforward implementation of the weighted sum procedure is often impractical, especially for problems that contain several competing objectives requiring trade-off consideration.

The second methodology relies on resolving directly the multiobjective problem by adopting a dedicated numerical optimization method. This approach finds optimal solution for the given set of objectives with an adapted definition of optimality. Multiobjective optimization aims at obtaining the set of solutions which satisfy the best trade-offs, or most suitable compromise, between the specified objectives. This set of optimal solutions define a surface in the parameter space, known as the Pareto front $(\mathrm{PF})$, or the set of non-dominated solutions in the objective space. A design $x$ is a Pareto optimal if there is no better design for the set of objectives. One common approach is to rely on on the development of Multiobjective Optimization Evolutionary Algorithms (MOEAs). ${ }^{25-31}$ MOEAS are able to produce the global set of optimal solutions in the form of the Pareto front exploring the parameter space using stochastic search to converge to the global solution. They have been inspired by biological evolutionary mechanisms, like mutation, selection, and crossover to broaden the search scheme during the optimization, improving the possibilities to converge to the global Pareto front.

There exists a wide variety of MOEAs able to deal with large parameter space both continuous and discrete parametrization. MOEAs include Genetic Algorithms (GAs), ${ }^{32-34}$ Covariance Matrix Adaptation Evolutionary Strategy (CMA-ES), ${ }^{35}$ and Particle Swarm Optimization (PSO). ${ }^{36-39}$ MOAEs have been extensively employed to optimize multifunctional electromagnetic problems in various disciplines. ${ }^{40-43}$ In the field of metasurfaces, multiobjective GAs have been used to optimize wavelength selective and absorbing metasurface designs. ${ }^{44}$ Advanced MOEAs have been developed for optimizing colour pixels metasurface ${ }^{45}$ and multi-resonant nanoridges for metasurface applications. ${ }^{46}$ Recently, a MOEA relying on 
BORG library ${ }^{30}$ has been exploited for generating a library of optimized freeform shapes to synthesize metasurfaces with multiple targets. ${ }^{47,48}$

Despite recent developments in MOEAs, there is still considerable concern about their practical use for realistic applications. Although MOEAs are capable of converging to the global set of solutions, MOEAs are computationally demanding and subject to high overheads iterations. This major limitation makes them impractical when combined with a costly threedimensional electromagnetic solver.

Recently, deep learning techniques focusing on multiobjective electromagnetic problems aare starting to impact on field of nanophotonics and metasurfaces. ${ }^{15,17,49-55}$ Deep learning substitutes the expensive simulations by a trained network able to predict the relationship between the set of parameters and the corresponding electromagnetic response. However, similarly as MOEAs, deep learning techniques require huge amount of simulations to train the network reliably. ${ }^{56,57}$ Novel approaches, known as generative models, ${ }^{57-59}$ have been adopted to accelerate the training process and have been utilized to optimize multifunctional nanophotonic devices. ${ }^{60-62}$ Despite this intense research effort, there is no yet a general relation defining the required number of simulations to train the network according to the number of objectives/parameters, indicating that multiple objective optimizations with training networks still require a considerable amount of resources. Besides, deep network is not inherently an optimization tool and suffers from convergence issues, notably in the case of competing objectives.

We adapted recently an alternative and efficient statistical learning optimization method, known as Efficient Global Optimization (EGO) ${ }^{63-65}$ for the design of single objective metasurfaces with a moderate budget of full-wave solver calls . ${ }^{66}$ This method utilizes a surrogate model to replace the high fidelity electromagnetic evaluation process with a simpler and cheaper model for the prediction of the new designs during the optimization process. EGO converges to the global solution with fewer iterations compared to classical global evolutionary strategies, making EGO an attractive optimization method for complex electromagnetic 
designs.

Here, we present a multiobjective optimization that relies on statistical learning. We demonstrate that this method converges rapidly to the global set of optimal solutions, using a moderate number of solver calls. We first discuss the main feature of the multiobjective EGO method, by providing a simple and clear illustration of its development. We then apply the multiobjective EGO to optimize the deflection efficiency of a Red, Green, and Blue (RGB) colors metasurface beam deflector. We show that despite the sophisticated competition between the three objectives, our optimization method is able to provide a single design based on a realistic configuration with more than $75 \%$ of average deflection efficiency for the three wavelengths. These results are depicted in the Supplementary information section.We then extend our analysis towards the optimization of a 3D RGB achromatic metalenses with moderate numerical apertures. Our numerical optimization study yields an average focusing efficiency of $45 \%$ using cylindrical nanopillars for a 3D metalens with a numerical aperture of 0.56 and diameter $\approx 10 \mu \mathrm{m}$. In addition, we confirm that the chromatic dispersion issue is diminished, and that the three wavelengths focus at the same position with a relative focusing error of $6 \%$. We experimentally confirmed the performances of the optimized designs, yieding a good agreement between numerical and experimental results.

\section{Multiobjective EGO}

To deal with expensive problems, it is common to resort to a surrogate model of the function of interest. The surrogate model provides a quick estimation to approximate the true function of interest to select the best candidates for the next simulation. Other alternatives such as support vector machines or neural networks, Gaussian process (GP) regression, also known as kriging, provides a probabilistic model. These modeling techniques are able to predict the value at n-evaluated locations while giving an estimate of the uncertainty about the prediction. It is crucial in optimization to balance exploration of poorly known regions 
of the input space (large predictive variance) with the exploitation of promising regions (low predictive mean). For more details about Bayesian optimization, we refer to Refs. ${ }^{67,68}$ The interested readers can also refer to our previous works ${ }^{16,17}$ where we describe the single objective EGO steps using simplified problems. In the following, we provide a simple illustration of the multiobjective EGO method which we are utilizing in the manuscript for multiwavelength metasurface optimization.

EGO method requires two phases: the first one, called the Design Of Experiment (DOE), aims at generating an initial database of designs which are evaluated using an electromagnetic solver to calculate all corresponding objective values. In the second phase, using the data obtained from the DOE, a Gaussian Process (GP) model is constructed for each objective, using an internal optimization process that fits the set of GP models to the DOE. The generated GP models for each objective provide a prediction of the objective values along with the parameter space. After achieving GP models for each objective, we determine its mean and the variance. The former corresponds to the expected values of the objectives at the given point in the design space, while the latter provides information about the uncertainty on these objective values, both providing the statistical information of the GP models. A statistical merit function is then established to identify which design, i.e. which set of parameters, should be tested in the next iteration to better approach the Pareto front. In our case, we consider the common criterion known as the Expected Hypervolume Improvement (EHI). ${ }^{69}$

The principle of the multiobjective optimization based on EGO using EHI selection criterion is described in Fig. 1. We consider here one parameter $X$ to optimize, and we aim at minimizing two distinct objectives. We assume 6 DOEs given by the blue points in Figs. 1(ab) together with the associated GP models (black curves). The red points in Fig. 1(c)), refer to non-dominated solutions (PF). Using GP models, we obtain information about the mean (value of the objective) and the uncertainty (variance) at each point in the design/objective space. The green and the cyan points indicate the predictive means (predictive values of the 

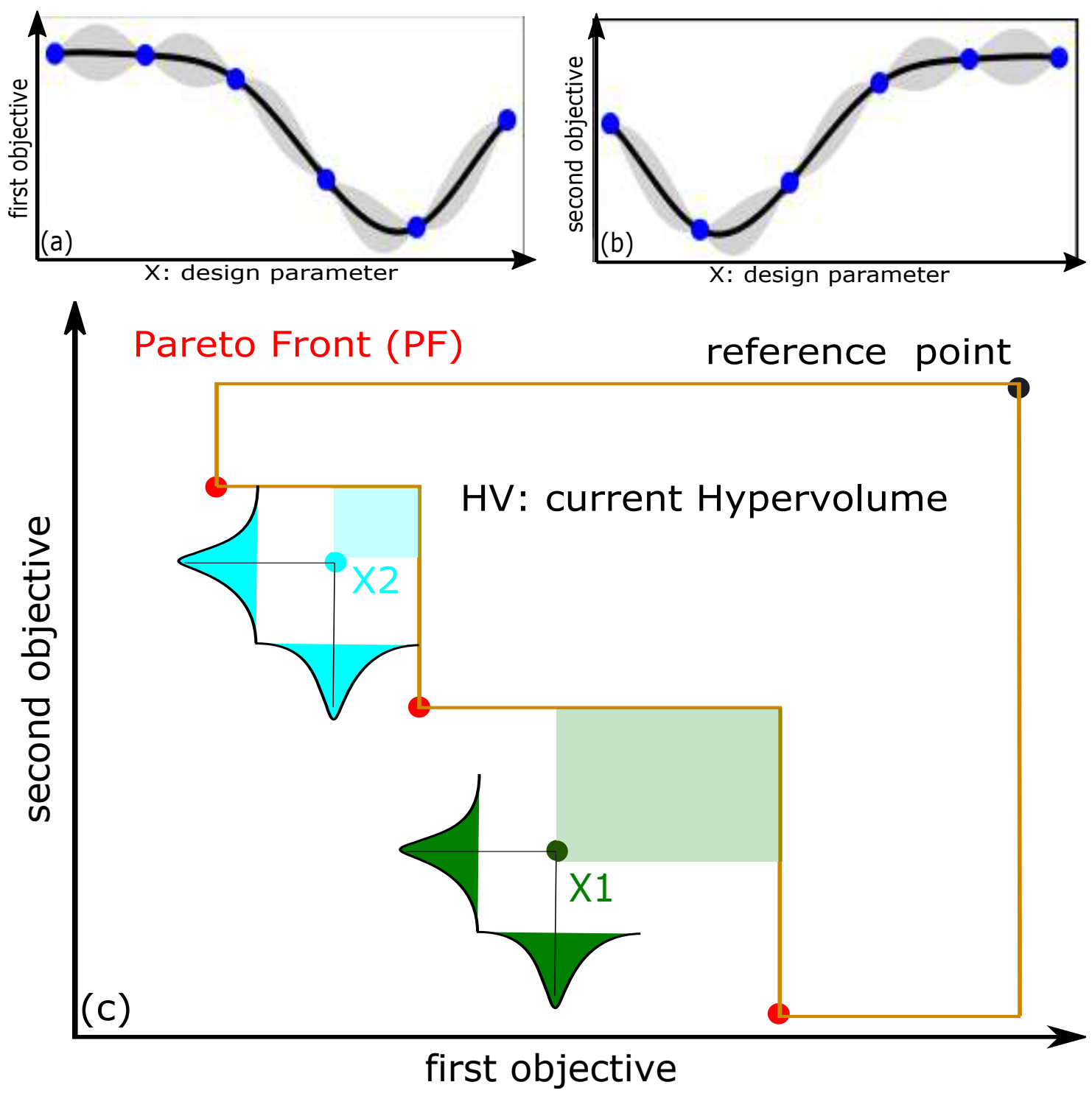

Figure 1: Illustration of the multiobjective optimization context and the expected hypervolume improvement (EHI) criterion. ${ }^{69,70}$ (a-b), two independent metamodels for the first and the second objective, respectively. The blue points refer to the DOEs and the grey curves represent the variance of each model. (c): objective space where the red points represent the existing non-dominated designs on the Pareto front $(\mathrm{PF})$. The orange region illustrates the current hypervolume (region between the current $\mathrm{PF}$ and a reference point). Dark green and cyan points refer to the predictive mean for two new designs $X_{1}$ and $X_{2}$, respectively. Shadow regions indicate the expected hypervolume improvement from $X_{1}$ and $X_{2}$. 
two objectives) for two novel designs $X_{1}$ and $X_{2}$, respectively. At this step, we are interested in choosing the design parameters to simulate next to converge faster to the exact Pareto front. We seek at computing the expectation of the hypervolume improvements (with a closed-form expression ${ }^{71}$ ) added to the current Pareto front (red points) from each Gaussian distribution around $X_{1}$ and $X_{2}$. One can see that the design $X_{1}$ is expected to increase the current hypervolume compared to the design $X_{2}$ as illustrated by the green and cyan shadow regions. We refer to Refs., ${ }^{70,72}$ for more details about the EHI selection criterion.

Considering our application metasurface context, we extract the parameter values corresponding to the maximized EHI in the parameter space. The corresponding design will be simulated using a fullwave electromagnetic solver. After that, the database is updated accounting for this new observation (construction of new GP models based on the updated database). This process is repeated until a predefined convergence criterion is reached, or when the EHI is sufficiently small. This specific statistical learning criterion makes our global optimization solver converges rapidly to the exact set of solutions. Besides, this feature demonstrates that EGO outperforms all the global evolutionary multi-objective optimizations and make it an ideal platform for time-consuming multi-functional nanoscale devices with competing objectives. In practice, we use the GPareto ${ }^{73,74} \mathrm{R}$ package to conduct multi-objective optimization.

We perform multiwavelength metasurface global optimization by coupling the above mentioned multi-objective EGO with a high-order fullwave electromagnetic solver (Discontinuous Galerkin Time-Domain, DGTD). ${ }^{75}$ DGTD can be viewed as a mixture of a classical (continuous) Finite Element Time-Domain (FETD) and the Finite Volume Time-Domain (FVTD) method, which turns out to be well adapted for the simulation of nanoscale light-matter interaction problems. ${ }^{76,77}$ The choice of time domain solver is fully justified for multi-wavelength metasurface designs, as all objective values (device performance in a wavelength range) are obtained with a single simulation run. Accounting for the full metasurface structure or elementary super cell unit, the solver is assessing near field coupling between the neighbouring 
elements, which is a fundamental factor in designing high efficient metasurfaces . ${ }^{16,78}$ Finally, due to the adaptability of our high-order DGTD solver in handling large-scale problems, the number of mesh cells is considerably reduced compared to other time-domain methods like the Finite-Difference Time-Domain (FDTD).

To perfect our optimization procedure for real case studies, we start optimizing a RGB beam steering metasurface. The results are shown in the supplementary information section. We then focus on large-scale RGB metalenses of pratical interest for imaging, able to focus light on RGB pixel sensors.

\section{Results and discussion}

\section{D large-scale RGB metalens}

In this section, we employ our methodology to the the design of a 3D achromatic metalens with high numerical aperture. Conventional approach relies on addressing phase profile to ensure focusing at a given fixed position $F$, according to:

$$
\phi(\omega ; y, y)=-\frac{\omega}{c}\left(\sqrt{x^{2}+y^{2}+F^{2}}-F\right)
$$

Here, $\omega$ is the angular frequency, $x, y$ represent the position of each individual element, and $c$ is the speed of light. Clearly, Eq. 1, is a frequency-dependent equation. To ensure high performance broadband focusing at a given fixed focal distance $F$, one has to control properly the chromatic dispersion of each scattering elements. The principal shortcoming of the classical synthesizing technique is the fact that phase dispersion is very sensitive to near field coupling, which could seriously degrade the focusing efficiency. Numerous 3D broadband

lenses have been optimized using the phase dispersion compensation procedure, ${ }^{79-83}$ and it is noticeable that the overall efficiency drops dramatically for lenses with $N A>0.5$, i.e. in regime for which the required spatial phase distribution change rapidly thus introducing 
strong difference in geometry between neighboring structures.

Optimizing the full metalens is another alternative manner to enhance the performance. This approach requires both a rigorous fullwave solver to account for the near field coupling and an efficient optimization algorithm. In the 3D case, few works have been published lately using freeform shapes optimization, ${ }^{84-86}$ yielding efficient devices but with sophisticated patterns that are difficult to manufacture. Other computational frameworks relying on gradient based architecture have been proposed in Refs. ${ }^{87-89}$ but they often do not reach the global designs.

It is worth mentioning that due to the simulation cost, optimization of full-metalenses have mostly been considered for 2D metalenses (cylindrical lens) , ${ }^{84,85,89-93}$ considering different optimization architectures and various numerical apertures. In the present work, we focus only on 3D spherical designs where the focusing is maintained along the three spatial dimensions at the visible regime.

Our work is distinct from what has been recently reported in the literature, where the metalens is either optimized via the synthesizing procedure or optimized based on freeform shapes building-blocks or with gradient-based strategies. First, we consider cylindrical shaped building-blocks which are easy to fabricate, and we take into account the fabrication constraints (achievable aspect ratio of the cylinder) which are usually hard to satisfy for most freeform optimized lenses. Second, we optimize almost all the meta-atoms and account for the strong near field coupling (optimizing as well the distances between nanostructures). Finally, we rely on a rigorous methodology by combining a global optimization method with a high-order DGTD solver to converge to the global solution, treating the large-scale problem with a moderate number of degrees of freedom.

In Tab. 1, we summarize results of recent works dealing with 3D achromatic metalens design (including our current work). For more details about the fundamental limitations of achromatic metalenses, we refer to Refs. ${ }^{94,95}$

We aim at optimizing a 3D metalens with NA between 0.45 and 0.6 that focus RGB 
Table 1: Comparison of 3D achromatic spherical metalens designs. Here ${ }^{(a)}$ refers to the experimental results, while ${ }^{(b)}$ is for the numerical simulation results.

\begin{tabular}{|l|l|l|l|l|}
\hline NA & Diameter $(\mu \mathrm{m})$ & Building blocks & Bandwidth & Average focusing efficiency \\
\hline 0.12 & 20.0 & Fishnet & $640-1200$ & $61^{(a)} \%^{82}$ \\
0.2 & 26.6 & Anisotropic unit cell & $460-700$ & $30^{(a)} \% 96$ \\
0.25 & 7.5 & Freeform & $480,650,850$ & $63^{(b)} \%^{84}$ \\
0.27 & 20.0 & Hybrid elements & $1000-1800$ & $60^{(a)} \%^{83}$ \\
0.26 & 6.0 & Cylinders & $400-700$ & $60^{(b)} \%^{93}$ \\
0.47 & 8.056 & Cylinders & $480,550,640$ & $55^{(b)} \% \& 44.16^{(a)} \%$ (this work) \\
0.56 & 10.22 & Cylinders & $480,550,640$ & $45^{(b)} \% \& 31.5^{(a)} \%$ (this work) \\
\hline
\end{tabular}

colors at the same focal plane with the maximum achievable efficiencies, seeking for the most suitable compromise between chromatic dispersion and efficiency, as depicted in Fig. 2(a)).

Our 3D metalens is composed of concentric rings of cylindrical nanopillars of GaN as depicted in Fig. 2(b). The height of the cylinders is fixed as 1000 nm. For the first design, we consider 12 concentric rings (apart from the central cylinder), in which the number of the cylinders in each ring (fixed during the optimization) increases when moving towards the outer rings. In general, we have roughly 407 cylinders in the whole metalens. The distribution of cylinders in each ring is summarized in Tab. 3 in the supplementary information.

To reduce both the computational cost and the number of optimization parameters, we rely on the symmetry properties of the $3 \mathrm{D}$ lens and consider only one-quarter of the structure as shown in Fig. 2(c) (a 3D view is presented in Fig. 9(a) in the supplementary information together with some details about the symmetry properties). Additionally, we assume that the cylinders in each individual ring share the same diameter to optimize only one row of cylinders along the radial direction and the distances between the rings, as indicated in Fig. 2(c) by the yellow cylinders and the black arrows, respectively. We also fixed the diameters of the cylinders in the first three rings together with the distances between the first seven rings. This is an admissible assumption since the relative phase profile (see Eg.1)) evolves slowly at the center Hence, one can arrange the distances between them to the minimum limit (in our case we choose $100 \mathrm{~nm}$ is the minimum feature size for the distance between rings). Moreover, the centre cylinder has a fixed diameter of $220 \mathrm{~nm}$ (based on our 

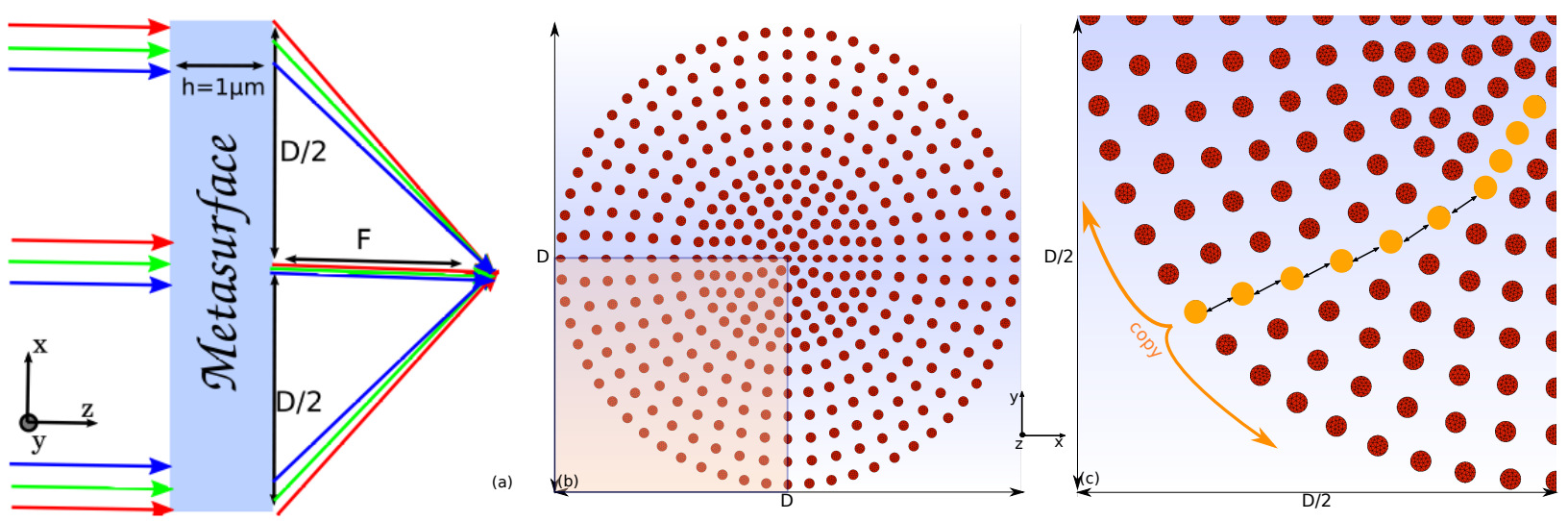

Figure 2: (a): schematic view of the 3D achromatic metalens in the $x-z$ plane. The metalens aims at focusing the three colors at the same focal distance $F$. The diameter of the metasurface is $D$ and the thickness is fixed as $h=1000 \mathrm{~nm}$. (b-c): $x-y$ plane for the 3D metalens under investigation, (b) for the whole metalens, and (c) for one-quarter of the geometry (due to the symmetry property) as illustrated by the shadow region in (b). The metalens consists of concentric rings of cylindrical GaN nanopillars (red region) placed on top of semi-infinite $\mathrm{Al}_{2} \mathrm{O}_{3}$ of dimension $10 \mu \mathrm{m} \times 10 \mu \mathrm{m}$, which is fixed during the optimization. The number of cylinders in each ring is given in the first two columns in Tab. 3. The 16 optimization parameters are depicted in (c), 10 diameters represented by the yellow cylinders and 6 parameters for the distances between the outer rings (black arrows).

former phase response calculations, ${ }^{16}$ this value is enough to give $2 \pi$ phase). In addition, the cylinders in the three sequential rings are assumed to have fixed diameters as $200 \mathrm{~nm}$ to ensure slow phase variation. In summary, in this configuration (Fig. 2(c)), we optimize only 16 parameters: 10 diameters represented by the yellow cylinders (vary between $90 \mathrm{~nm}$ and $200 \mathrm{~nm}$ ) and 6 distances between the outer rings indicated by the black arrows (vary between $100 \mathrm{~nm}$ and $250 \mathrm{~nm}$ accordingly with nanofabrication capabilities).

Note that with our parametrization, the diameter of the lens is slightly varying based on the position of the last ring, indicating that the NA is different for all 3 wavelengths during the optimization. For all 3 wavelengths we target a fixed focal distance $F$ during all the iterations of $7.5 \mu \mathrm{m}$. For this first design, we are maximizing the power at the focal plane $(z=7.5 \mu \mathrm{m})$ for the blue color at $\lambda=480 \mathrm{~nm}$, green color at $\lambda=550 \mathrm{~nm}$, and for the red color at $\lambda=640 \mathrm{~nm}$. For normalization of the focusing efficiency during the optimization process, we calculate the power at the focal plane to the power passing through 

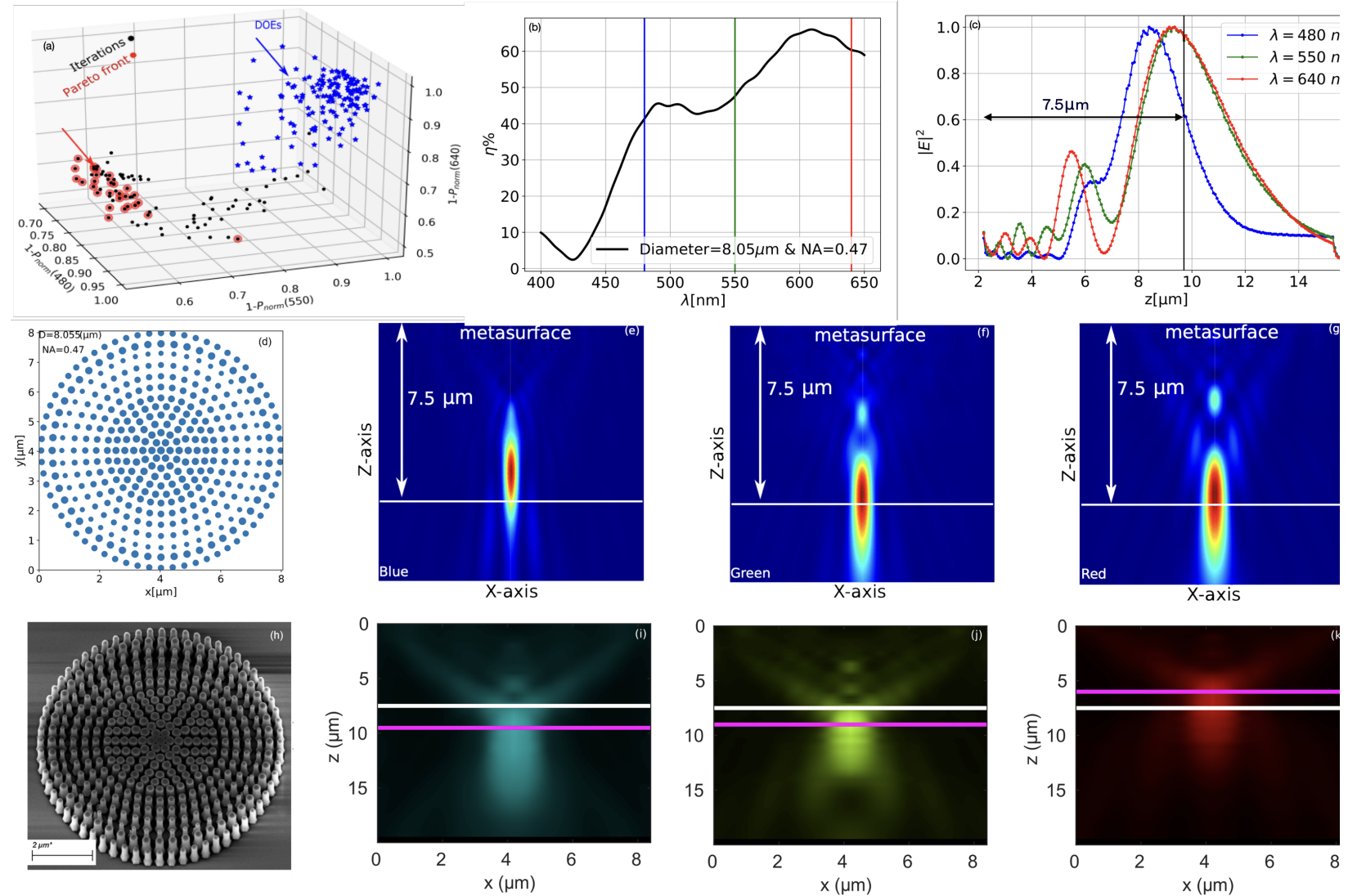

Figure 3: (a): optimization results for the first metalens design with 16 optimization parameters, composed of 12 concentric rings as shown in Fig. 3(d). The blue points refer to the 90 designs in the DOE database, the black ones indicate the optimization iterations. The red points represent the Pareto front (see the text for more details). The diameter of the chosen lens is targeting around $8 \mu \mathrm{m}$ (the associated parameters are given in Tab 3). (b) We chose one of the best designs along the Pareto front (red arrow in (a)), where the three objectives are maximized simultaneously and extract the numerical focusing efficiency as a function of the wavelength. The efficiency is calculated as the ratio of the power at the focal plane to the power passing through an aperture of diameter $8.055 \mu \mathrm{m}$ (diameter of the optimized lens). The three white vertical lines refer to the three wavelengths considered during the optimization. The numerical convergence is studied in Fig. 9(b). (c): cut along $z$ at the center of the lens for the RGB colors. The vertical line refers to the target focusing position F. (d): indicate the $\mathrm{x}-\mathrm{y}$ profile of the optimized design. (e-g): numerical intensity profiles at the $x-z$ plane. (h): Scanning electron micrographs (SEM) of the fabricated lens and (i-k) illustrate the experimental focusing intensity at the $x$ - $z$. The intensity is measured using closest available LED sources emitting at $\lambda=490 \mathrm{~nm}, \lambda=550 \mathrm{~nm}$, and $\lambda=660 \mathrm{~nm}$ for the blue, green, and red colors, respectively. 
a circular aperture of diameter $10 \mu \mathrm{m}$ (fixed during the optimization process). In our case, the focusing efficiency is estimated as the ratio between the power in the focal plane (circle or radius $800 \mathrm{~nm}$ ) divided by the power passing through a circular aperture with diameter $D$. After optimization, we correct diameter of the optimized design and we calculate the exact focusing efficiency and compute the actual numerical aperture. It is worth mentioning that the diameter of the focal plane is fixed for the three wavelengths to be three times the average of the theoretical full width at half maximum (FWHM) of the three wavelengths. This might introduce small bias for the focal distance, however, as we shall see below, the overall bias is minimal. Based on the above procedure and the given fixed focal distance $F=7.5 \mu \mathrm{m}$, the NA is varying roughly between 0.32 and 0.6 .

In Fig. 3(a), we present the optimization results for the first lens with 12 concentric rings. The normalized power is denoted as $P_{\text {norm }}$. We are considering a minimization problem, such that the main objectives are to minimize $1-P_{\text {norm }}$ for $\lambda=480 \mathrm{~nm}, \lambda=550 \mathrm{~nm}$, and $\lambda=640 \mathrm{~nm}$. In this particular example, we optimize 16 parameters for satisfying 3 objectives, considering a DOE database with 90 elements (blue points) for the initial learning process. These points are then used to construct 3 metamodels (one for each objective) and use the EHI criterion to select the next design to be simulated. The optimization iterations are outlined by the black points in Fig. 3(a) and the design along the Pareto front are indicated by the red points. This figure shows that only 180 iterations are sufficient to capture the Pareto front (the convergence results are depicted in Fig. 10 in the supplementary information section). The optimized lens, obtained by electing the best point along the Pareto front, is shown in Fig. 3(d). We have found that the optimized design yields a metalens with diameter $D=8.055 \mu \mathrm{m}$, with a numerical aperture of $N A=0.473138$. Accordingly, we computed the power passing through a circular aperture with this $D=8.055 \mu \mathrm{m}$ as a reference power to estimate the actual focusing efficiency of the metalens, see Fig. 3(b) where the associated values are $42 \%, 49 \%$, and $60 \%$ for the blue, green, and red colors, respectively.

Fig. 3(c) refers to the cut along $z$ direction. This results is used to estimate a relative 
focusing error along $z$ of about $4 \%$ error for the green and red colors, and approximately $13 \%$ for the blue, see Figs. 3(e-g).

The optimized metalens design has been fabricated using nanofabrication techniques on GaN semiconductor grown using metal-organic chemical vapor deposition on sapphire substrate. The final device is shown on the SEM image in Fig. 3(h). The numerical optimized designs are realized accordingly to nanofabrication limitation with maximum aspect ratio of 10 (ratio between the smallest diameter and the height of the cylinders). If better designs are achievable numerically, their constraints yield a challenging fabrication and are not considered further. The fabrication process is provided in the supplementary information section. The fabricated lens has been characterized using the experimental setup given in Fig. 12 in supplementary information section (see Ref. ${ }^{97}$ for more details). The x-z experimental intensity maps are depicted in Figs. 3(i-k), where the average relative focusing error is estimated as 19\% (horizontal white line for the predefined focal length, to be compared to the actual focal length denoted by the purple line). The tendency of the focal lengths are in good agreement with the numerical results, except for the blue light for which the calculated focal length is shorter than the expected one (Figs. 3(e)) while it is longer for the measurements (Figs. 3(i)). This discrepancy is related to fabrication imperfections, i.e., deviations from the design diameter of the pillars, and have more impact on the device characteristics for shorter wavelengths. To quantitatively compare the numerical and experimental results, we estimated the experimental focusing efficiencies of the lens at the three wavelengths as $45,6 \%, 39.3 \%$ and $47,6 \%$ for blue, green and red colors, respectively, with an average focusing efficiency estimated as $44.16 \%$. The experimental focusing efficiencies have been estimated as the ratio between the power at the focal plane within an aperture of a diameter defined by the FWHM of the experimental focusing spot and the power impinging on an aperture defined by the diameter of the lens (see more details in Supplementary Information). The measured and numerical focusing efficiencies have the same tendency as a function of the wavelength. However, the measured efficiencies are slightly lower than the numerical ones 
which is attributed to the fabrication imperfections. Note that the experimental focal spots are broader than the numerical spots (see Fig. 13 in the supplementary information section). This is due to the fact that the experimental and numerical focal spots are determined differently. The numerical focal spot corresponds to the diffraction limited spot or, equivalently, point spread function (PSF) of the lens. It is determined by considering a plane wave (point source at the infinity) as an incident light beam. However, the experimental focusing spot corresponds to the image of the considered source in our experiments. Its size is determined by the extent of the diaphragm D2 of the illumination system as depicted in Fig. 12 in the supplementary information section.

In the following, we present a study to further improve the obtained results by adding supplementary rings to the optimized design in order to increase the NA and improve the control over the dispersion to better reduce the chromatic aberrations.

In order to further promote the focusing performance of the optimized lens shown in Fig. 3(d), we propose a supplementary step as illustrated in Fig. 4(a). The optimization process is necessary to capture complex near-field coupling between adjacent nanostructures, but it does not affect the response of far distant elements. It is therefore possible to optimize the lens section-by-section, keeping the first 11 rings of metastructures optimized from the previous step, and increasing the size of the component by appending three additional rings to the outer part. The outermost ring in Fig. 3(d) is relaxed and will be combined with the additional three rings as shown in Fig. 4(a). The shadow region indicates the optimized part of the former design. While the diameters of the four highlighted cylinders indicate the optimization parameters together with the distances between the additional rings symbolized by the black arrows. Similar to the previous design, all cylinders in the same ring are chosen to share the same diameter. Obviously to keep coherence with the first optimized lens section, we consider optimization at a fixed focal distance of $7.5 \mu \mathrm{m}$.

Increasing the diameter of the lens increases the phase mismatch between the three 

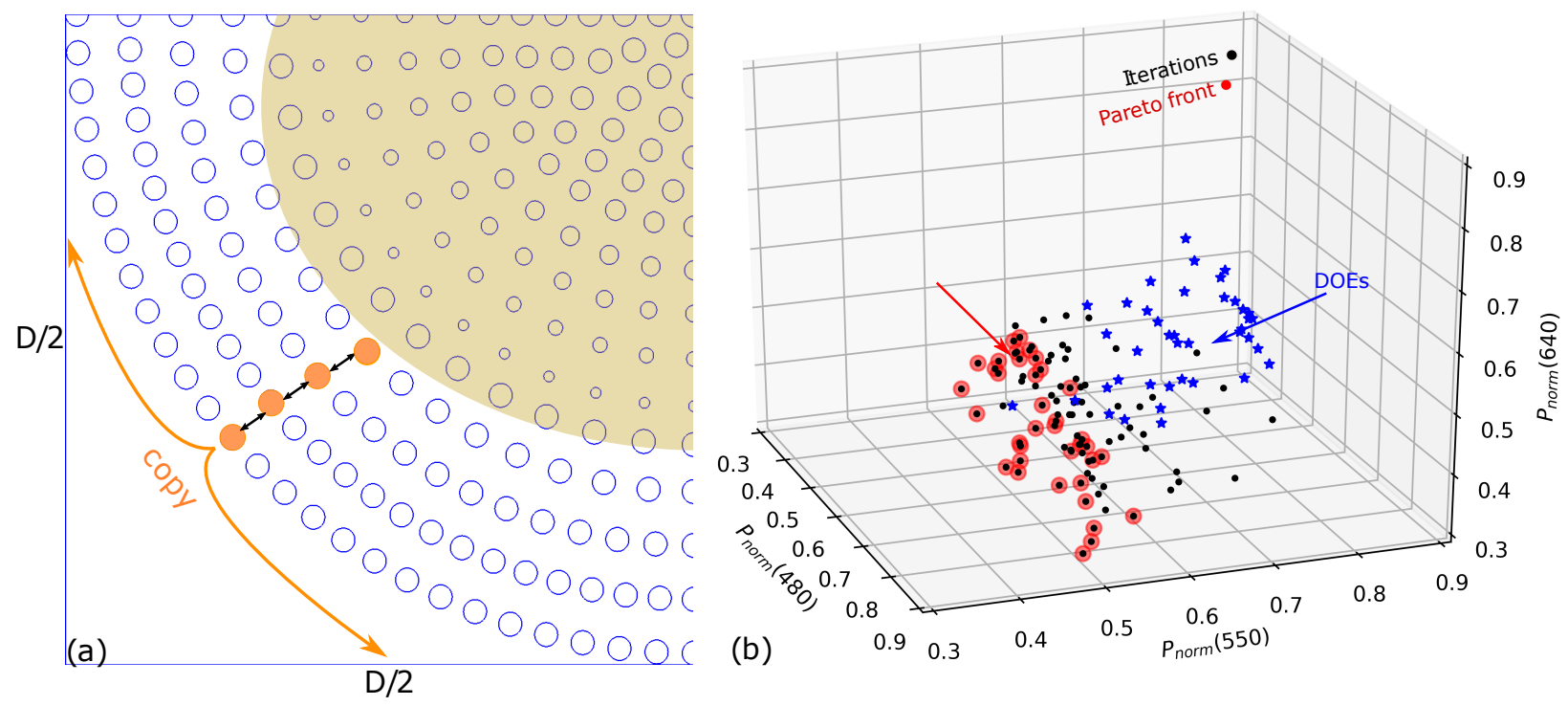

Figure 4: Extended metalens with a larger diameter and a reduced set of parameters. (a): sketch of the extended diameter lens, where the central part is fixed from previous optimized design given in Fig. 3(d) as it is represented by the shadow region. We relax the diameter of the last ring in Fig. 3(d), and add 3 more rings. In total we optimize only 7 parameters; 5 diameters (orange cylinders) and three other parameters for the distances between the outer rings (black arrows). (b): illustrates the optimization results in the second case. Here we considered 50 DOEs (blue points) and 150 iterations (black points). The Pareto front is illustrated by the red points, i.e., the set of non-dominated designs. The red arrow, refers to the results presented in Fig. 5. 
wavelengths, and thus imposing constrains with higher dispersion compensation. It also increases the numerical aperture. However, this method of optimization by sections reduces the computational cost to 7 parameters only instead of 25 for the full lens with the extra rings. The optimization results are given in Fig. 4(b), where the DOE, optimization iterations, and Pareto front are given in blue, black, and red colors, respectively.

As previously, we chose one of the best middle points along the Pareto front. The corresponding design yields a lens of diameter $D=10.22 \mu \mathrm{m}$. This diameter is slightly larger than the one obtained from the previous design. Moreover, the numerical aperture becomes 0.56 instead of 0.47 for the previous device (the fabricated lens is exhibited in Fig. 5(c)). Based on this diameter $d=10.22 \mu \mathrm{m}$, we numerically estimated the focusing efficiency of the lens. As illustrated in Fig. 5(a), the computed focusing efficiencies are almost 43\%, 41\%, and $47 \%$ for the blue, green, and red colors, respectively. To the best of our knowledge, this is the highest focusing efficiency obtained for RGB colors for a $3 \mathrm{D}$ metalens with $\mathrm{NA}=0.56$ (see Tab. 1), especially designed using classical nanopillars that are considerably easier to fabricate with respect to complex freeform geometries. We would like to mention that increasing the NA increases the phase dispersion needed to achieve multiwavelength behavior, which thus slightly decreases the overall average focusing efficiency of the device.

Moreover, the cut along $z$-axis provided in Fig. 5(b) unitedly with the numerical intensity profiles represented in Figs. 5(d-f) reveal that the chromatic dispersion effect is diminished compared to the previous lens with relative focusing error for the blue, red colors is $6.1 \%$, and $0.5 \%$ for the green. This relative error of $6.1 \%$ could be due to the bias introduced from the definition of the output plane, as we argued above. Interestingly, our experimental results depicted in Figs. 5(g-i) confirms the decrease of the chromatic dispersion compared to the previous results presented in Fig. $3(\mathrm{i}-\mathrm{k})$. The average measured focusing efficiency is approximately $31.5 \%$ (28\%, 27.3\%, 39.1\% for blue, green and red respectively).

It has been demonstrated that by reducing the numerical aperture, it is possible to increase the focusing efficiency with a numerical aperture below $0.2 .{ }^{82}$ However, for a 3D 

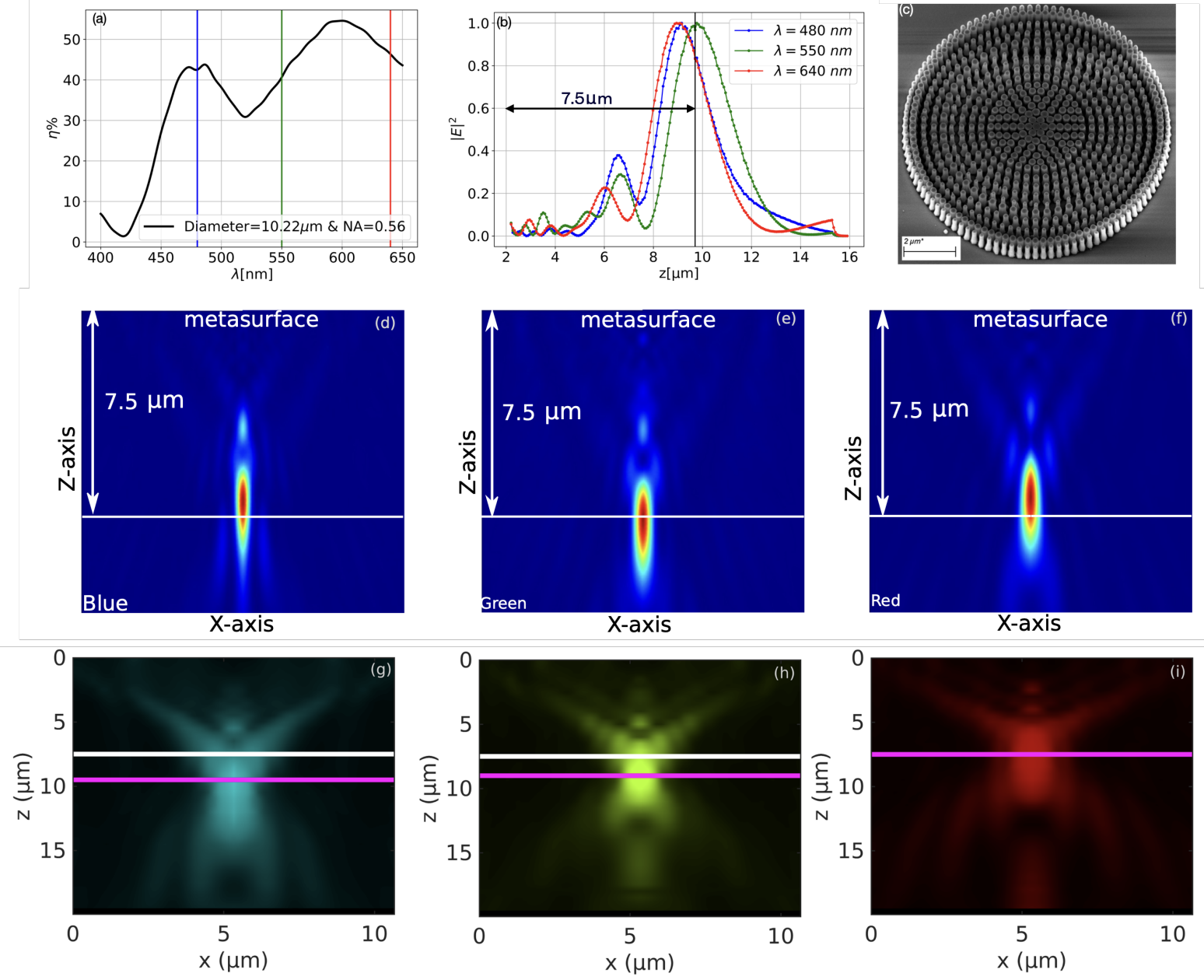

Figure 5: Optimization results for the extended design with 7 optimization parameters, composed of 15 concentric rings shown in Fig. 4(a). The results corresponds to one of the best design along the Pareto front (red arrow in Fig. 4(b)), where the three objectives are maximized simultaneously. The diameter of this lens is $10.22 \mu \mathrm{m}$. The full set of parameters are given in Tab. 4 in the supplementary information section. (a): provides the focusing efficiency of the optimized lens calculated as the ratio of the power at the focal plane to the the power passing through an aperture of diameter $10.22 \mu \mathrm{m}$. The three vertical lines refer to the three wavelengths considered during the optimization. (b): cut along $\mathrm{z}$ at the center of the lens for the RGB colors. The vertical line refers to the target focusing position along $\mathrm{z}(z=7.5 \mu \mathrm{m})$. The fabricated lens is depicted in $(\mathrm{c})$, besides, the corresponding intensity profiles at the $x-z$ plane is given in (g-i), where the intensity is measured at $\lambda=490 \mathrm{~nm}$, $\lambda=550 \mathrm{~nm}$, and $\lambda=660 \mathrm{~nm}$ for the blue, green, and red colors, respectively. The numerical focusing intensity for each color are drawn in (d-f). 
lens fabricated using cylindrical nanopillars, it is challenging to compensate the chromatic dispersion and maintain high power efficiency for a numerical aperture above 0.5. One can extend this work to further increase the numerical aperture by changing either the material/shape of the building blocks, or even introduce new degrees of freedom to further control the dispersion.

\section{Conclusion}

In this work, we apply a global statistical multiobjective optimization to the design of multiwavelength metasurfaces. In addition to achieving global optimal design, this method converges using an adequate number of iterations, unlike the traditional multiobjective optimization techniques. Despite the inherent competing nature of the objectives, our optimization method can provide a single design based on a realistic configuration with more than $75 \%$ of average deflection efficiency with only 150 iterations (see supplementary information section). These unique features make our optimization method an ideal candidate for optimizing various multifunctional metasurface configurations.

We extend our optmization to the design of multiwavelength spherical metalens that operate at the visible regime. The main objective was to focus the RGB colors at the same focal plane and maximize the focusing efficiency for the three colors. In this study, we aim at optimizing spherical metalens with $N A>0.5$ using classical cylindrical nanopillars with the highest possible focusing efficiency.

Unlike the conventional modelling technique, we optimized nearly all the nanoresonators, considering relatively long range near field coupling. We optimized different lenses with different numerical apertures. The first design consists of 13 concentric rings leading to a lens with diameter $\approx 8 \mu \mathrm{m}$ and a numerical aperture of 0.47 with $55 \%$ average numerical focusing efficiency and $13 \%$ focal error. We then extended our method of optimization, enlarging the devices with sequentially optimized sections by adding 4 supplementary rings 
to the initial optimized design. Our enlarged numerical optimization study yields an average numerical focusing efficiency of $45 \%$ using cylindrical nanopillars with a numerical aperture of 0.56 (diameter $\approx 10 \mu \mathrm{m}$ ) and confirmed that the chromatic dispersion issue is diminished with a relative focusing error of $6 \%$ for the three wavelengths.

Furthermore, we fabricated and characterized experimentally the optimized lenses and found a good agreement between the numerical and the experimental results with an average measured focusing efficiency of $44.16 \%(N A=0.47)$ and $31.5 \%(N A=0.56)$. To the best of our knowledge, this is the highest focusing efficiency obtained in such spherical metalens configuration with $N A>0.5$.

This combination of full-wave numerical solver with the multiobjective EGO method offers an ideal platform for optimizing multifunctional metasurfaces. Various device of practical interest, including RGB lenses, color holograms, multifunctional polarization-dependent metasurfaces and other multi-wavelength optical components could benefit from this approach. Realizing multiobjective optimal design is certainly the key to promote metasurface technology to real-life applications.

\section{Acknowledgement}

The authors acknowledge support from French defence procurement agency under the ANR ASTRID Maturation program, grant agreement number ANR-18-ASMA-0006-01. Numerical experiments presented in this work have been conducted on two HPC (High Peformance Computing) resources: on one hand, the Occigen supercomputer of CINES under the re-

source allocation 2020-A0080610263 made by GENCI and, on the other hand, the PlaFRIM experimental testbed, supported by Inria, CNRS (LABRI and IMB), Université de Bordeaux, Bordeaux INP and Conseil Régional d'Aquitaine. 


\section{Supporting Information Available}

\section{Optimizing of RGB light deflector}

As a state of the art illustration, we employ the multiobjective EGO in an attempt to optimize a beam steering metasurface at three different wavelengths simultaneously. We aim at designing a highly efficient Red, Green, and Blue (RGB) light deflector based on a realistic configuration. In order to realize this task, we design a metasurface composed of rectangular Gallium Nitride (GaN) on top of a semi-infinite $\mathrm{Al}_{2} \mathrm{O}_{3}$ substrate (see red and green regions in Fig. 6(a), respectively). We consider five rectangular nanopillars in a supercell, with a period corresponding to $1500 \mathrm{~nm}$ along $y$ direction, while along $x$ direction, we chose a period of 190 $\mathrm{nm}$ to limit the diffraction in the substrate, especially for short wavelengths. The height of the rectangular pillars is fixed as $h=1000 \mathrm{~nm}$. In this study, a normal incident plane-wave with electric field polarized along $y$ axis is injected from the substrate. The width of each rectangular nanopillar along $x$ and the distance between the neighboring ridges are kept fixed during the optimization as $100 \mathrm{~nm}$ and $90 \mathrm{~nm}$, respectively. In order to control the phase dispersion in the $y-z$ plane, we vary the length of the rectangular nanopillars along $y$ (represented by the white arrows in Fig. 6(a)). It is worth mentioning that the minimum length of the ridges is considered as $90 \mathrm{~nm}$, which corresponds to minimum feature size achievable by nanofabrication of $\mathrm{GaN}$ with height $=1000 \mathrm{~nm}$.

The main objective is to maximize the light deflection efficiency for the first order mode (in the $y$ - $z$ plane) for the RGB colors. We define three different wavelength intervals for each color in which we aim at optimizing the average efficiency $\left(\eta_{\text {avg }}\right)$. The intervals for blue, green and red colors are, respectively, $(450-480) \mathrm{nm},(520-550) \mathrm{nm}$ and $(620-650) \mathrm{nm}$. Thus, in our optimization framework (minimization problem), we consider five parameters (width of each pillar along $y)$ and three objectives (1- $\eta_{\text {avg }}\left(\right.$ Blue), $1-\eta_{\text {avg }}($ Green $)$, and $1-\eta_{\text {avg }}($ Red $)$ ).

As we have explained in the main text, the primary step in the EGO method is to construct the design of experiments (DOEs) database. In this particular example, we have 

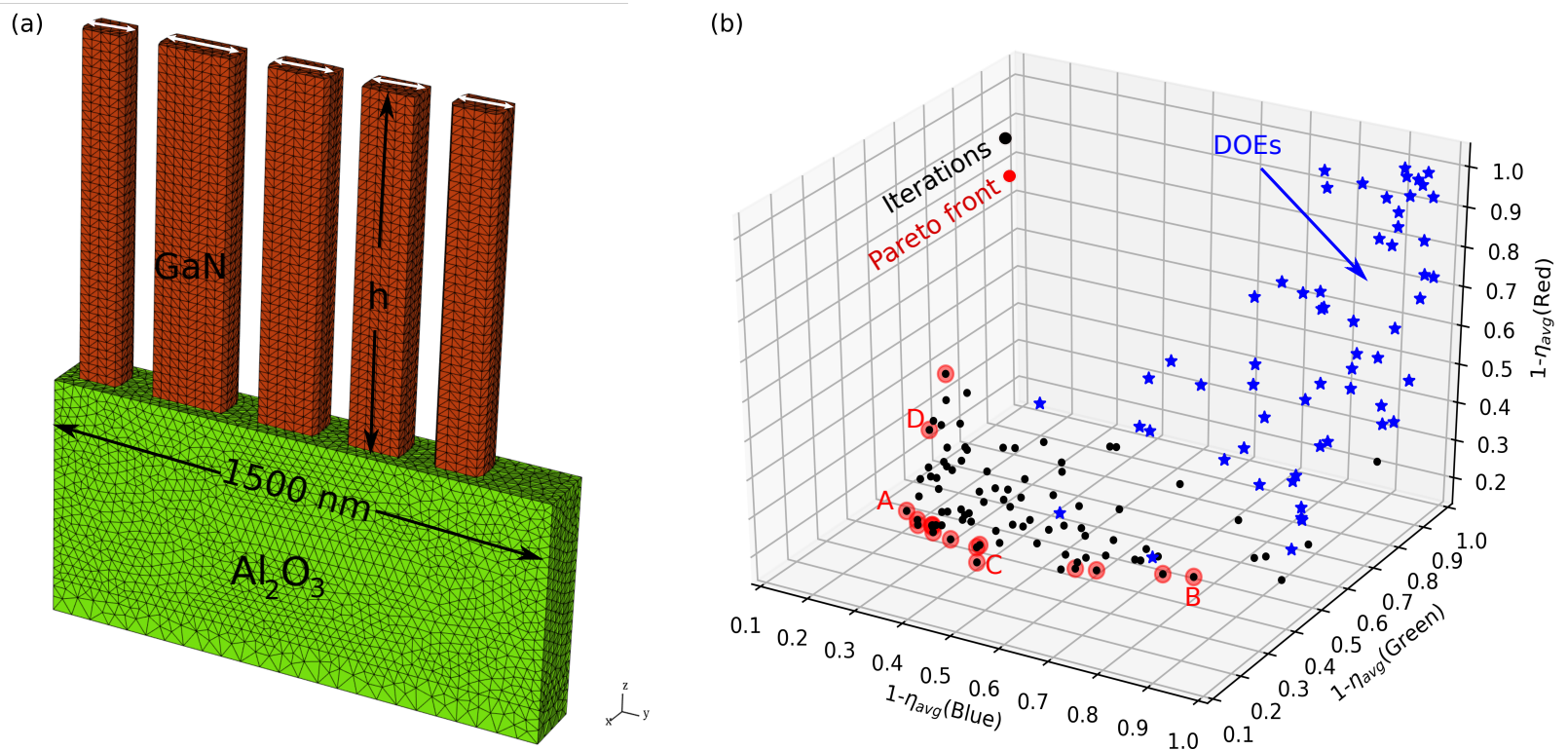

Figure 6: Optimization results for the RGB light deflector. (a): geometry under consideration, composed of five rectangular nanopillars (red regions) of GaN placed on top of a semi-infinite substrate made of $\mathrm{Al}_{2} \mathrm{O}_{3}$ (green region). The height of the pillars is fixed as $h=1000 \mathrm{~nm}$, their widths along $x$ are also fixed as $100 \mathrm{~nm}$. The period of the super-cell along $y$ is $1500 \mathrm{~nm}$, while along $x$ is associated to $190 \mathrm{~nm}$. The optimization parameters are represented by the white arrows (widths of the pillars along $y$ direction). (b) indicates the optimization results, where the blue points refer to the 60 DOEs. The black points are for the optimization iterations (90 points), while the red circles indicate the Pareto front. A-D letters refer to 4 designs along the Pareto front that will are considered in Fig. 7.

considered 60 elements in the DOE database (the corresponding objective function values are depicted as blue points in Fig. 6(b)). Based on this database, three GP models are generated for each objective accompanied by an expected hypervolume improvement (EHI) criterion to identify the next design for each iteration (see Fig. 1 in the main text manuscript and its associated text). The selected design (given by specific values of the set of parameters) is then simulated with our DGTD fullwave solver.

The performed optimization iterations are represented by the black points in Fig. 6(b)) while the red points identify the set of non-dominated points in the objective space (Pareto front). The most striking result that emerges from these data is that 90 optimization iterations (black points) are enough to capture the Pareto front. This peculiarity of the multiobjective EGO method makes it a viable alternative to the classical heuristic evolu- 
tionary algorithms where a considerable number of iterations are required to approximate the Pareto front. ${ }^{47}$ The discussion about the convergence of the considered metamodels is elaborated in the next section of this document (see Fig. 8 and its associated text)
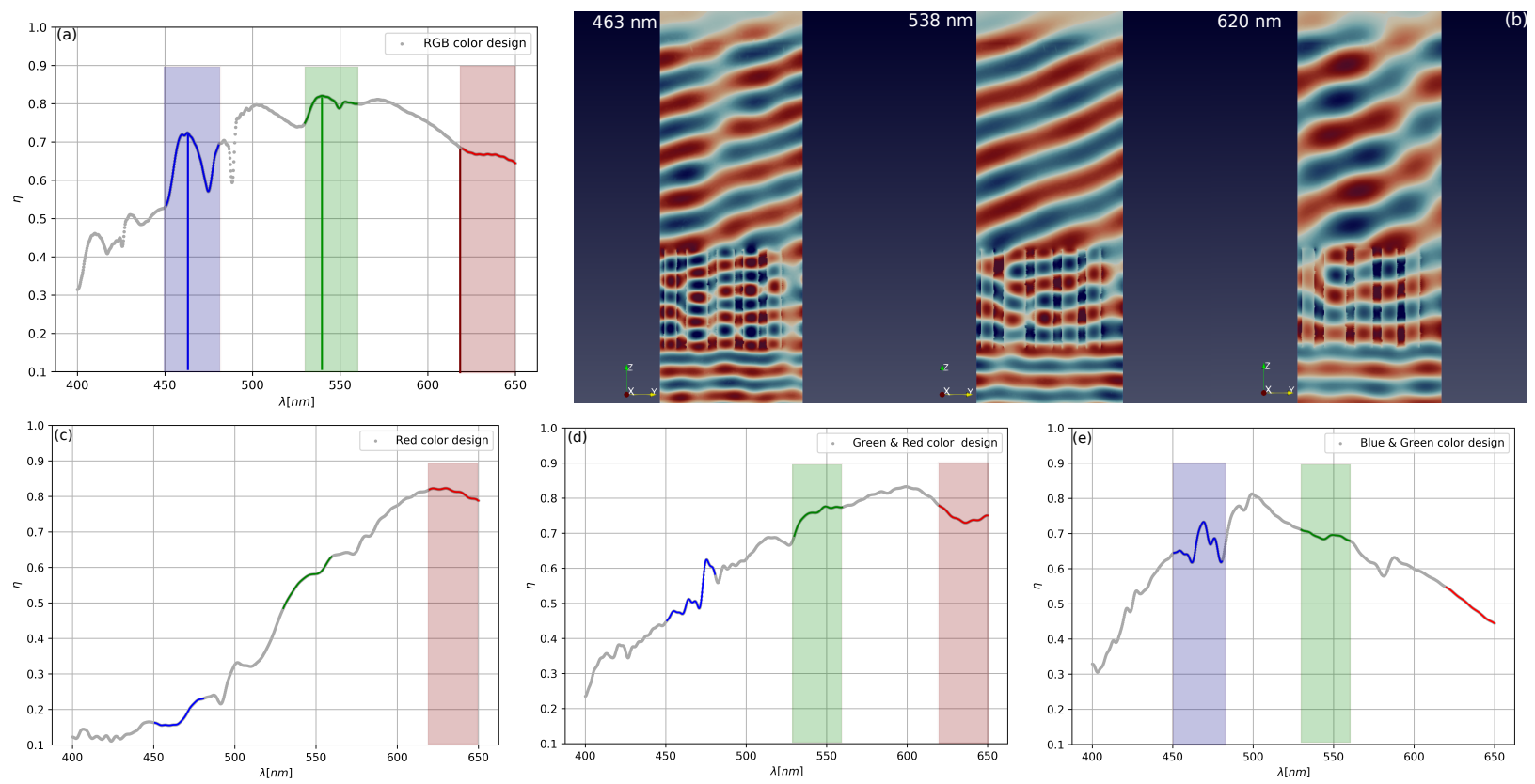

Figure 7: (a-b): results for the best middle point along the Pareto front shown in Fig. 6(b). (a): the deflection efficiency for the first order mode is represented by the gray curve as a function of the wavelength. The blue, green, and red parts on the gray curve denote the optimization interval defined for each color. The vertical color lines, refer to the wavelengths where the efficiencies attain their maximum in each interval. (b) $\Re e\left(E_{y}\right)$ at the maximum efficiency in each interval. (c-e), optimization results for various designs along the Pareto front shown in Fig. 6(b). (c): refers to the optimized design for the red color, (d), for the green and red colors, and finally (e) for the blue and green colors. The corresponding optimization parameters are given in Tab. 2.

It is worth highlighting that the most beneficial attribute of any multiobjective optimization is the construction of the Pareto front, which materializes the trade-off between the competing objectives. In Fig. 7, we present different optimized designs. Figs. 7(a-b) refers to a middle point along the Pareto front (point A in Fig. 6(b)) where all the objectives are optimized simultaneously; we refer to this design as the RGB design (the associated optimized parameters are given in the second column in Tab. 2). In Fig. 7(a), we plot the deflection efficiency of the first order mode for the RGB design. As it can be seen, the 
mean deflection efficiencies for the three regions are enhanced concurrently, and the average efficiency reaches $65.5 \%, 80.0 \%$, and $66.5 \%$ for blue, green, and red intervals, respectively. The vertical lines indicate the wavelengths where the maximum efficiency is achieved in each interval $(72 \%, 82 \%$, and $69 \%$ deflection efficiencies at wavelengths of $463 \mathrm{~nm}, 538 \mathrm{~nm}$, and $620 \mathrm{~nm}$, respectively). The corresponding field profiles are plotted in Fig. 7(b) where the light bending is adequately presented for the three wavelengths.

The most notable observation that emerges from the field profile comparison in Fig. 7(b) is the ability of the optimized nanoridges to treat the different longitudinal modes for the considered wavelengths. This peculiar treatment results in an average effective mode index profile for each wavelength to maximize the deflection efficiency for three wavelengths at the same time. Remarkably, this correlation is related to strong near field interactions between the elements, as it is depicted in Fig. 7(b). As can be appreciated, these interactions are different for the three wavelengths and they depend on the number of longitudinal modes that propagate in each element for each wavelength. This confirms that our optimization method identifies the set of parameters that satisfy the three objectives taking into account the strong near field coupling between the ridges. This appealing results cannot be achieved using the classical phase synthesizing approach where each ridge is placed based on its phase and transmission response for a given wavelength. Besides, these results further strengthened the viability of our numerical methodology by combining a high-order fullwave solver with an efficient global optimization method.

In Figs. 7(c-e), we present different optimized designs along the Pareto front (points CD in Fig. 6(b), respectively) to further extend the discussion and provide the reader with more insights about the usefulness of the multiobjective optimization. Figs. 7(c-e), refer to optimized designs where we focus on the red color, both the green and red colors, and finally both the blue and green colors, respectively. The corresponding optimization parameters are given in Tab. 2. These results emphasize the significant trade-off between the three objectives. We note that more than $80 \%$ of deflection efficiency can be achieved if one focuses 
Table 2: Optimization parameters for a four designs presented in Fig. 7(a) and Figs. 7(ce), for the RGB, Red, Green-Red, and Blue-Green colors, respectively. The height of the nanoridges is fixed as $h=1000 \mathrm{~nm}$, and the widths along $x$-direction are fixed as $100 \mathrm{~nm}$. The distances between ridges are fixed as $90 \mathrm{~nm}$. Besides, the period along $x$ is given as 190 $\mathrm{nm}$, while along $y$ is $1500 \mathrm{~nm}$.

\begin{tabular}{|l|l|l|l|l|}
\hline Width $(\mathrm{nm})$ & RGB & Red & Green-Red & Blue-Green \\
\hline $\mathrm{w}_{1}$ & 127.96 & 249.51 & 129.42 & 236.92 \\
$\mathrm{w}_{2}$ & 233.19 & 216.14 & 246.70 & 189.76 \\
$\mathrm{w}_{3}$ & 177.71 & 169.21 & 179.26 & 162.52 \\
$\mathrm{w}_{4}$ & 158.48 & 113.48 & 145.79 & 148.30 \\
$\mathrm{w}_{5}$ & 142.72 & 127.78 & 142.15 & 136.52 \\
\hline
\end{tabular}

only on a single objective as it is the case in Fig. 7(c), while the efficiency drops when two competing objectives are considered, as expected (see Figs. 7(d-e)). Nevertheless, thanks to our global multiobjective EGO method, an adequate balance between the three objectives is obtained as discussed previously (Figs. 7(a-b)). To the best of our knowledge, these results demonstrate the highest RGB light deflector efficiency based on realistic metasurface configuration.

\section{Convergence of the RGB deflector design}

In Fig. 8 (a-c), we study the leave-one-out predictions versus true values for each GP models, one for each objective, respectively. In these figures, each point corresponds to the prediction values from each metamodels as if the point was removed from the training set, compared to the exact value. In other words, for all the points along the line (for each sub figure), the predicted values deduced by the metamodels are equal to the true values. Obviously, we are more interested in low values (minimization problem), where the fitting deduced by the three models are in a good agreement with the exact values. Besides (data not shown), the EHI for the last 30 iterations remains of the order of $10^{-2}$ which is another indication that 90 iterations are enough to approximate the Pareto front as given in Fig. 7(b). 

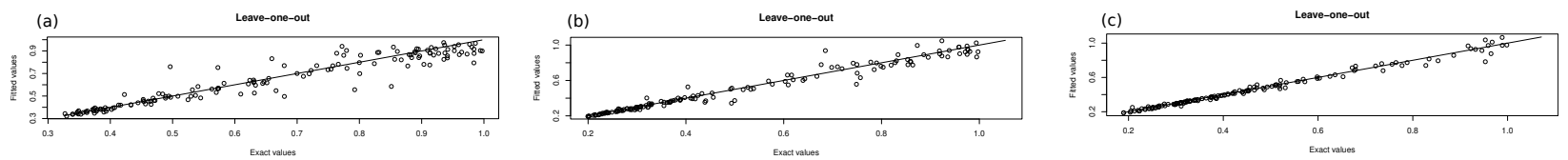

Figure 8: Illustration of the optimization convergence for RGB light deflector results presented in Fig. 6(b)

\section{Additional information for the metalens}

In this section, we provide additional data to complement our numerical results presented in the main manuscript.

In Fig. 9(a) we provide additional information about the symmetry properties. Besides, in Fig. 9(b) we demonstrate the convergence of one of the optimized lenses.

In Fig. 10 we discuss the optimization convergence of the first lens presented in Fig. 3 in the main manuscript. The associated parameters can be found in Tab. 3. Tab. 4 refers to the optimization parameters for the results presented in Fig. 5 in main manuscript.

In Fig. 11, we further demonstrate the versatility of our numerical methodology mutually with our fabrication abilities. We imitate another design along the PF shown in Fig. 4 (b). In this special case, we focus solely on optimizing the red color. As it can be seen in Fig. 11 (a), the focusing efficiency is roughly $67 \%$, while it is dramatically reduced for the other colours. The enhanced focusing efficiency can be understood from the cut along z-axis drawn in Fig. 11 (b), where only the red colour is focused at the desired focal plane (see also the field maps in Figs. 11 (d-f)). Thanks to our fabrication platform, the fabricated design presented in Fig. 11 (c), only focuses the red color at the desired focal plane as evidenced in Figs. 11 (g-i) and the measured focusing efficiency for the red color is $\approx 44 \%(\approx 25 \%$ for the blue and $\approx 22 \%$ for the green as expected from the numerical results). The above results represent another proof to validate both the numerical and the experimental results obtained.

Finally, we discuss, in the folowing paragraphs, further information for the fabrication procedure and the experimental characterization setup Fig. 12 and results Fig. 13 of the fabricated lenses. 

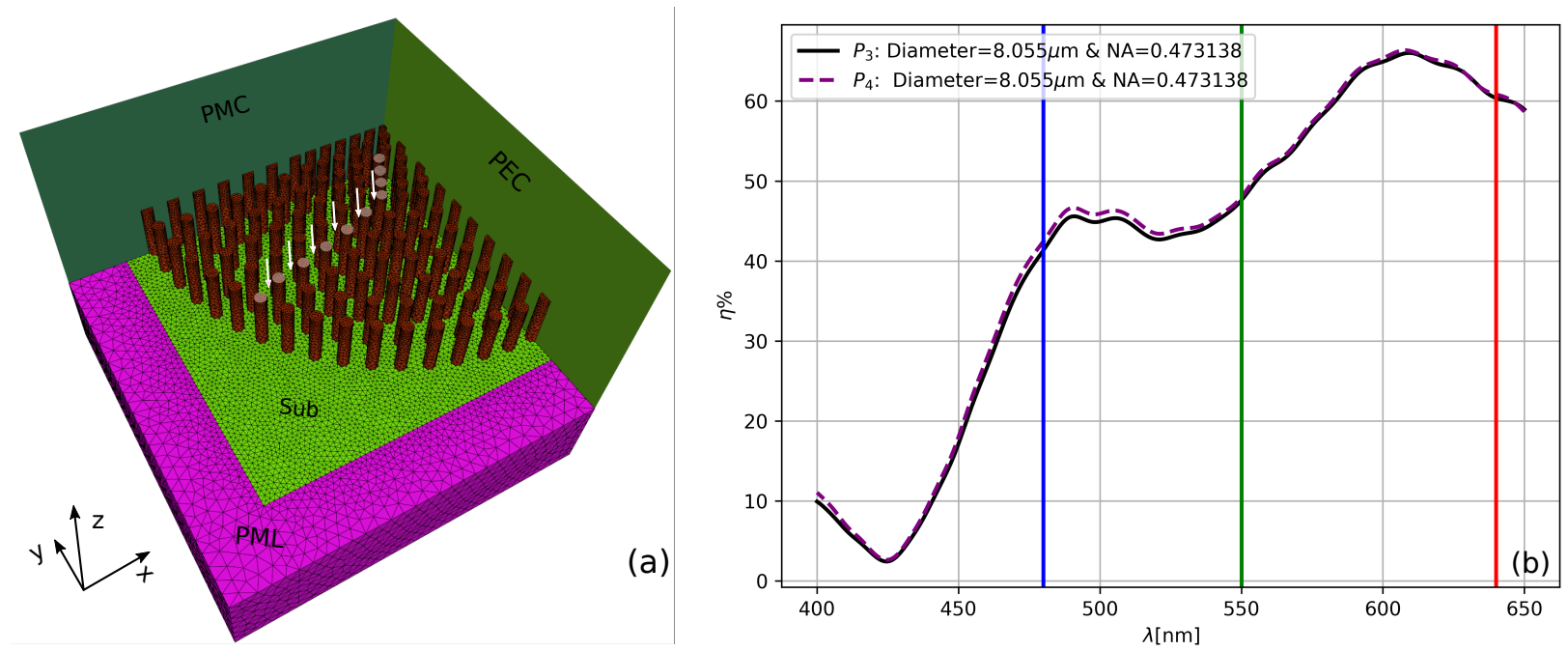

Figure 9: (a): 3D Schematic view for one quarter of 3D metalens under investigation (see Fig. 2 for the $x-y$ view). The red cylinders are made of GaN and the substrate is composed of $\mathrm{Al}_{2} \mathrm{O}_{3}$. The optimized cylinders are highlighted in white circles along with the distance between the outer rings (white arrows). We consider a normally incident plane wave from the substrate with electric field polarized along $x$-axis. Therefore, in order to mimic the full geometry, we consider a perfect electric conducting (PEC) condition on the right most faces (where the electric field is oscillating) and perfect magnetic conducting (PMC) along the front face. During the optimization iterations, we consider a mesh of size corresponding to 5 points per wavelength inside the cylinders. In general, the number of cells is almost 800,000. In the framework of our DGTD fullwave solver, we consider a third order polynomial $P_{3}$ interpolation to approximate the electromagnetic fields, which is enough to get accurate solutions during all the optimization iterations. The justification is shown in (b) where the agreement between the results obtained for $P_{3}$ and $P_{4}$ interpolations (for the results presented in Fig. 3) is fully demonstrated. Each iteration takes almost $1 \mathrm{~h}$ using 720 cores. The numerical experiments presented in this paper were carried out using the PlaFRIM experimental testbed, supported by Inria, CNRS (LABRI and IMB), Université de Bordeaux, Bordeaux INP and Conseil Régional d'Aquitaine. 

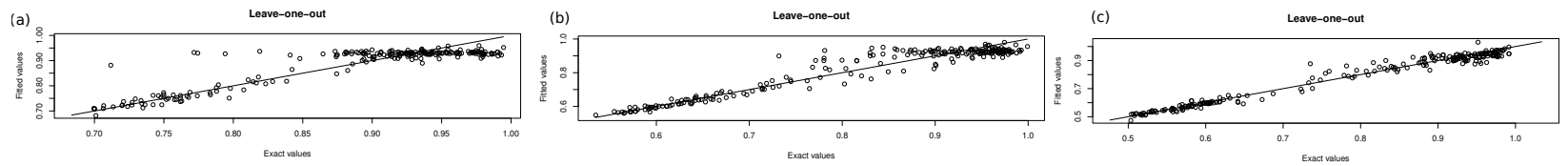

Figure 10: Illustration of the optimization convergence for the results presented in Fig. 3. Here we study the leave-one-out predictions versus true values for each GP models. (a) for first objective, (b) for the second objective, and (c) for the third objective. In these figures, each point corresponds to the prediction, if the point was removed from the training set, compared to the exact value. In other words, for all the points along the line, the predicted values deduced by the metamodels are equal to the true values. A subtlety is that all values are still used to estimate the parameters of the GP models and not removed. Hence they do influence the results in an indirect way. Plateaus around one at the top (especially for the first two objectives) could indicate that a large part of the design space has this value (see blue points in Fig. 3(a)). However, flat regions are hard to model for GPs, hence worsening prediction. Fortunately here we are more interested in low values (maximize the the efficiency in this minimization problem), that are better fitted for the three models.

Table 3: Optimization parameters for the metalens presented in Fig. 3 with diameter $D=$ $8.055 \mu \mathrm{m}$ and $\mathrm{NA}=0.473138$. The first two columns refer to the number of rings (first) and the associated number of cylinders (second). The third column gives the diameter of the cylinders in each ring. The fourth column indicates the distances between rings (for instance, $\operatorname{Ring}_{2,1}$ refers to the distance between the second and the first rings). Their values are given in the last column.

\begin{tabular}{lllll}
\hline Ring & cylinders & Radius $(\mathrm{nm})$ & Distance indicators & Distance values in $\mathrm{nm}$ \\
\hline Central & 1 & 110.000 & - & - \\
1 & 7 & 100.000 & $\operatorname{Ring}_{1, c e n t}$ & 100.000 \\
2 & 13 & 100.000 & $\operatorname{Ring}_{2,1}$ & 100.000 \\
3 & 15 & 100.000 & $\operatorname{Ring}_{3,2}$ & 100.000 \\
4 & 21 & 94.6262 & $\operatorname{Ring}_{4,3}$ & 100.000 \\
5 & 27 & 91.3074 & $\operatorname{Ring}_{5,4}$ & 100.000 \\
6 & 33 & 85.7489 & $\operatorname{Ring}_{6,5}$ & 100.000 \\
7 & 37 & 79.9027 & $\operatorname{Ring}_{7,6}$ & 188.312 \\
8 & 41 & 70.8879 & $\operatorname{Ring}_{8,7}$ & 191.255 \\
9 & 45 & 62.1650 & $\operatorname{Ring}_{9,8}$ & 216.618 \\
10 & 49 & 45.0000 & $\operatorname{Ring}_{10,9}$ & 250.000 \\
11 & 53 & 100.000 & $\operatorname{Ring}_{11,10}$ & 120.437 \\
12 & 65 & 69.5238 & $\operatorname{Ring}_{12,11}$ & 192.588 \\
\hline
\end{tabular}


Table 4: Optimization parameters for the extended metalens presented in Fig. 5 with diameter $D=10.22 \mu \mathrm{m}$ and $\mathrm{NA}=0.56$. The first 11 rows are identical to the the ones in Tab. 3 (the meaning of the columns remains the same). The green color refers to the optimized rings in the extended design.

\begin{tabular}{lllll}
\hline Ring & cylinders & Radius $(\mathrm{nm})$ & Distance indicators & Distance values in $\mathrm{nm}$ \\
\hline Central & 1 & 110.000 & - & - \\
1 & 7 & 100.000 & $\operatorname{Ring}_{1, \text { cent }}$ & 100.000 \\
2 & 13 & 100.000 & $\operatorname{Ring}_{2,1}$ & 100.000 \\
3 & 15 & 100.000 & $\operatorname{Ring}_{3,2}$ & 100.000 \\
4 & 21 & 94.6262 & $\operatorname{Ring}_{4,3}$ & 100.000 \\
5 & 27 & 91.3074 & $\operatorname{Ring}_{5,4}$ & 100.000 \\
6 & 33 & 85.7489 & $\operatorname{Ring}_{6,5}$ & 100.000 \\
7 & 37 & 79.9027 & $\operatorname{Ring}_{7,6}$ & 188.312 \\
8 & 41 & 70.8879 & $\operatorname{Ring}_{8,7}$ & 191.255 \\
9 & 45 & 62.1650 & $\operatorname{Ring}_{9,8}$ & 216.618 \\
10 & 49 & 45.0000 & $\operatorname{Ring}_{10,9}$ & 250.000 \\
11 & 53 & 100.000 & $\operatorname{Ring}_{11,10}$ & 120.437 \\
12 & 65 & 68.0160 & $\operatorname{Ring}_{12,11}$ & 192.588 \\
13 & 105 & 51.5016 & $\operatorname{Ring}_{13,12}$ & 250.000 \\
14 & 105 & 90.5210 & $\operatorname{Ring}_{14,13}$ & 228.898 \\
15 & 105 & 61.0380 & $\operatorname{Ring}_{15,14}$ & 111.765 \\
\hline
\end{tabular}

Table 5: Optimization parameters for the lens given in Fig. 11 with diameter $D=10.66 \mu \mathrm{m}$ and NA $=0.5755$. The first 11 rows are identical to the the ones in Tab. 3 (the meaning of the columns remains the same). The green color refers to the optimized rings in the extended design.

\begin{tabular}{lllll}
\hline Ring & cylinders & Radius $(\mathrm{nm})$ & Distance indicators & Distance values in $\mathrm{nm}$ \\
\hline Central & 1 & 110.000 & - & - \\
1 & 7 & 100.000 & $\operatorname{Ring}_{1, c e n t}$ & 100.000 \\
2 & 13 & 100.000 & $\operatorname{Ring}_{2,1}$ & 100.000 \\
3 & 15 & 100.000 & $\operatorname{Ring}_{3,2}$ & 100.000 \\
4 & 21 & 94.6262 & $\operatorname{Ring}_{4,3}$ & 100.000 \\
5 & 27 & 91.3074 & $\operatorname{Ring}_{5,4}$ & 100.000 \\
6 & 33 & 85.7489 & $\operatorname{Ring}_{6,5}$ & 100.000 \\
7 & 37 & 79.9027 & $\operatorname{Ring}_{7,6}$ & 188.312 \\
8 & 41 & 70.8879 & $\operatorname{Ring}_{8,7}$ & 191.255 \\
9 & 45 & 62.1650 & $\operatorname{Ring}_{9,8}$ & 216.618 \\
10 & 49 & 45.0000 & $\operatorname{Ring}_{10,9}$ & 250.000 \\
11 & 53 & 100.000 & $\operatorname{Ring}_{11,10}$ & 120.437 \\
12 & 65 & 87.209 & $\operatorname{Ring}_{12,11}$ & 192.588 \\
13 & 105 & 45.000 & $\operatorname{Ring}_{13,12}$ & 250.000 \\
14 & 105 & 100.000 & $\operatorname{Ring}_{14,13}$ & 250.000 \\
15 & 105 & 88.443 & $\operatorname{Ring}_{15,14}$ & 202.365 \\
\hline
\end{tabular}



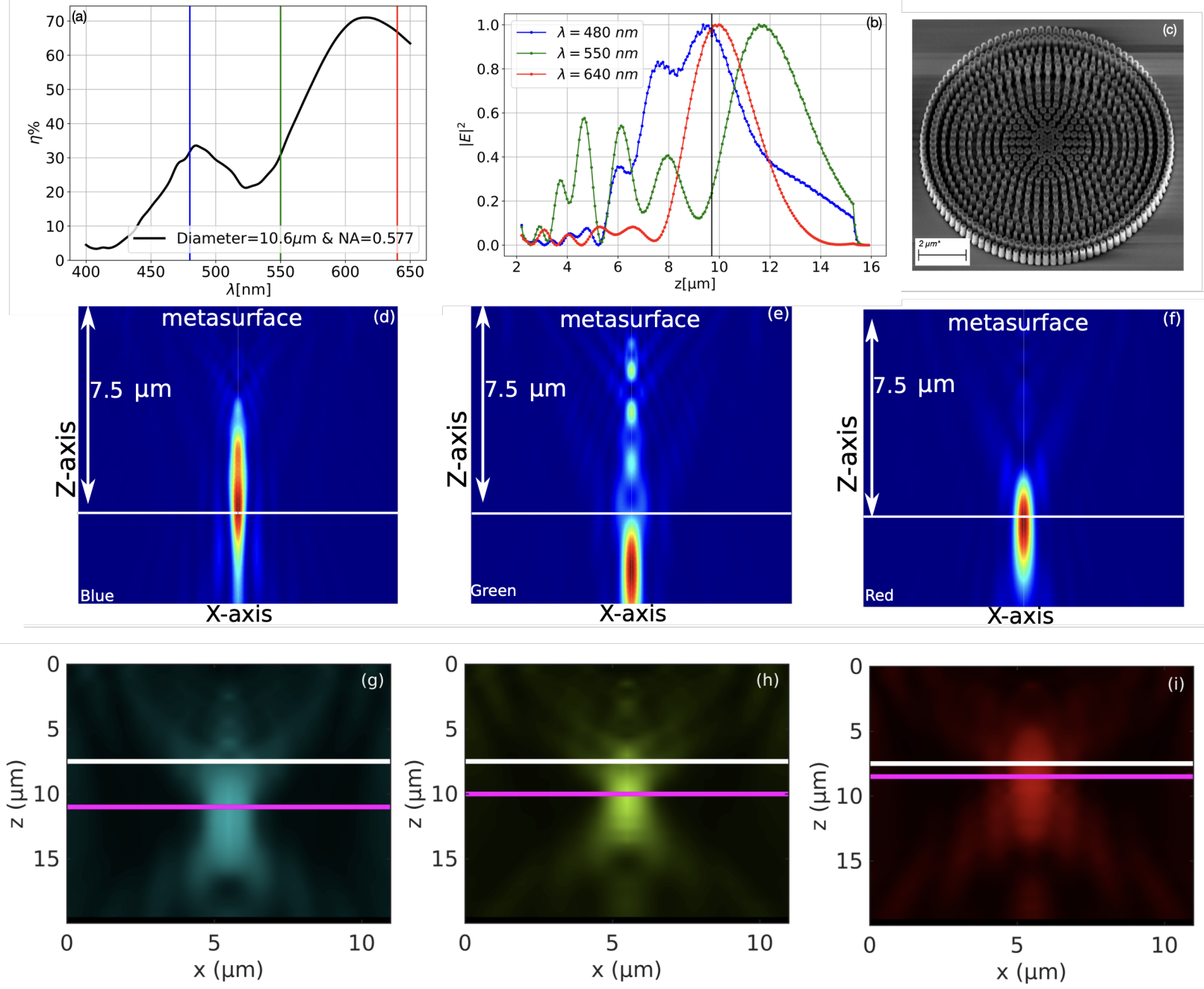

Figure 11: Optimized design for focusing the red color. The diameter of this lens is $10.66 \mu \mathrm{m}$ as indicated in. Besides, the full set of parameters are given in Tab. 5. (a): provides the focusing efficiency of the optimized lens, where it is maximized for $\lambda=640$ (approximately $69 \%$ ). (b): cut along $\mathrm{z}$ at the center of the lens for the RGB colors. The vertical line refers to the target focusing position along $\mathrm{z}(z=7.5 \mu \mathrm{m})$, where the red color achieves its maximum (c): represents the fabricated lens. (d-f): numerical intensity profiles along $x-z$ plane for the three optimized wavelengths. (g-i): refer to the experimental intensity profiles for the three colors. 


\section{Fabrication process}

The metalenses were made of GaN pillars with $1 \mu \mathrm{m}$ in height. They have been revealed by patterning a $1 \mu \mathrm{m}$ thick GaN layer grown on a c-plan sapphire substrate using a metalorganic chemical vapor deposition. Conventional electron beam lithography (EBL) was used to expose a double layer of $\approx 200 \mathrm{~nm}$ PMMA resist (495A4) spin-coated on the GaN thinfilm and then baked on a hot plate at $125^{\circ} \mathrm{C}$. E-beam resist exposure was then performed at $20 \mathrm{keV}$ (using a Raith ElphyPlus, Zeiss Supra 40), followed by PMMA development using a 3:1 IPA:MIBK solution. After development, a $50 \mathrm{~nm}$ layer of $\mathrm{Ni}$ was deposited using e-beam evaporation to perform a metallic film liftoff by immersing the sample into acetone solution for $2 \mathrm{~h}$. The resulting Ni pattern was utilized as a hard mask during the reactive ion etching (RIE, Oxford system with a plasma composed of $\mathrm{Cl} 2$, CH4, Ar gases, with flows of 13, 2, and $2 \mathrm{sccm}$, respectively) to transfer the pattern in the GaN layer. Finally, the Ni hard mask on the top of GaN nanopillars was removed using chemical etching with a 1:2 $\mathrm{HCl} / \mathrm{HNO} 3$ solution.

\section{Characterization setup}

To characterize the fabricated metalenses, we used a home-made inverted optical microscope as depicted in Fig. 12. The illumination part consists of LEDs centred at three different wavelengths (490, 550 and $660 \mathrm{~nm})$. The LEDs are positioned in Köhler optical scheme in order to illuminate the sample with controlled spot size (diaphragm D1) and numerical aperture (Diaphragm D2). The light passing through the metalens sample is collected by a microscope objective positioned on moving stage to detect different planes along the optical axis $\mathrm{Oz}$ (perform z-scan). It is worth-noting that in this configuration the focal plane of the metalens is conjugated with the plane of the diaphragm D2. The light is then sent to the tube lens and finally to the camera detector (see ref [S.Khadir et al. ACS Photonics 2021] for more information). 


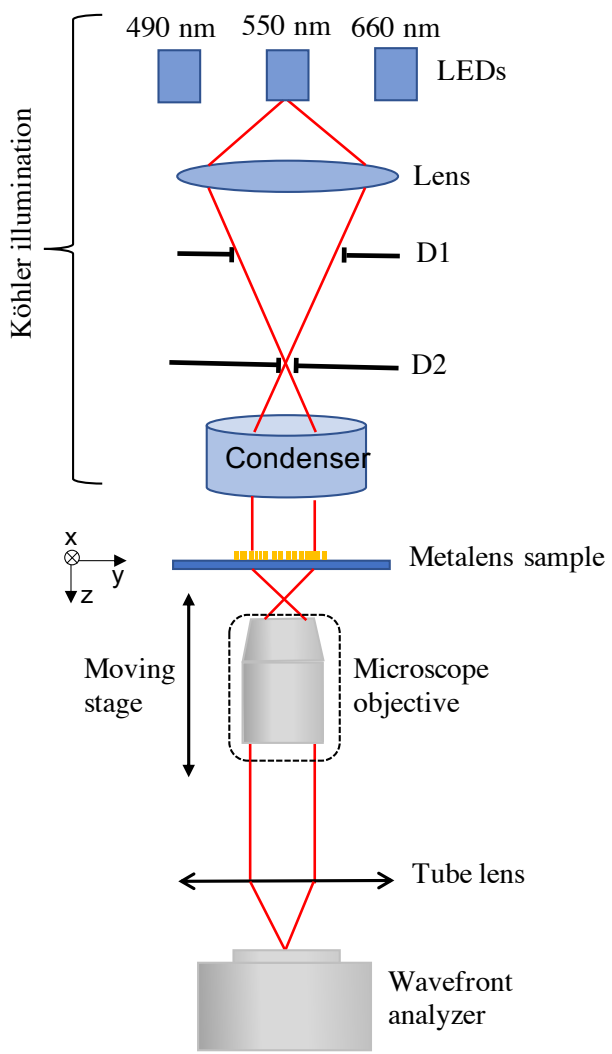

Figure 12: Scheme of the optical setup used to characterize the metalenses. LEDs with different wavelengths combined to a Köhler configuration illuminates the sample with a light beam controlled in wavelength, size, and numerical aperture. The light passing through the metalens sample is collected by a microscope objective lens and sent to the detector. The microscope objective is mounted on moving stage to perform z-scan of the light passing through the metalens
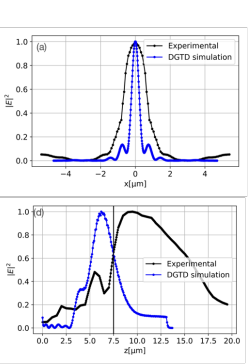
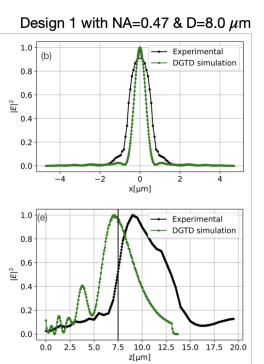
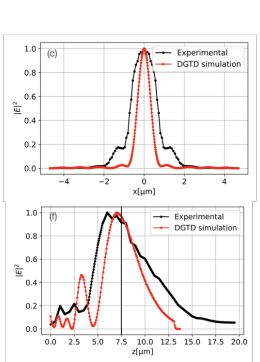
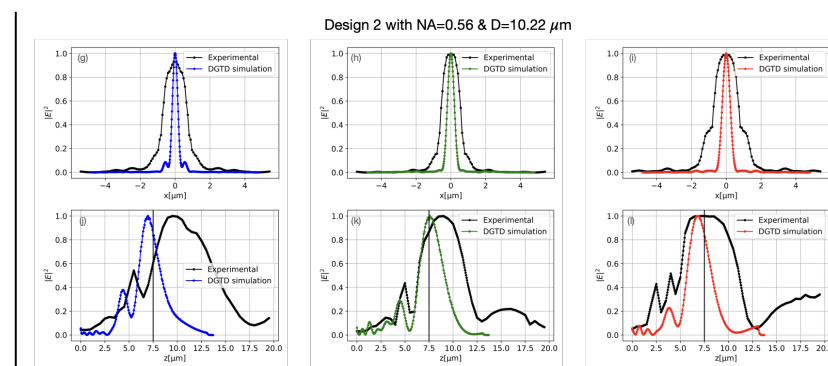

Figure 13: Comparison of the experimental and numerical intensity profile cuts for the first metalens design (Fig. 3 of the main text) (right side) and the second design (Fig. 5 of the main text) (left side). The first row corresponds to the $1 \mathrm{D}$ cut along $\mathrm{x}$ axis at the focal distance $F=7.5 \mu \mathrm{m}$. One can see the broadning of the experimental focal spots in comparison with numerical ones as discussed in the main text. The second row corresponds to the cut along $\mathrm{z}$ axis at the center of the lens showing a quite good agreement of the focusing distance between experiments and simulations. See the main text manuscript for further explanations. 


\section{References}

(1) Astilean, S.; Lalanne, P.; Chavel, P.; Cambril, E.; Launois, H. High-efficiency subwavelength diffractive element patterned in a high-refractive-index material for $633 \mathrm{~nm}$. Optics letters 1998, 23, 552-554.

(2) Lalanne, P.; Astilean, S.; Chavel, P.; Cambril, E.; Launois, H. Design and fabrication of blazed binary diffractive elements with sampling periods smaller than the structural cutoff. JOSA A 1999, 16, 1143-1156.

(3) Yu, N.; Genevet, P.; Kats, M. A.; Aieta, F.; Tetienne, J.-P.; Capasso, F.; Gaburro, Z. Light Propagation with Phase Discontinuities: Generalized Laws of Reflection and Refraction. Science 2011, 334, 333-337.

(4) Sun, S.; He, Q.; Xiao, S.; Xu, Q.; Li, X.; Zhou, L. Gradient-index meta-surfaces as a bridge linking propagating waves and surface waves. Nature materials 2012, 11, 426.

(5) Lin, D.; Fan, P.; Hasman, E.; Brongersma, M. L. Dielectric gradient metasurface optical elements. Science 2014, 345, 298-302.

(6) Lee, M.-S. L.; Lalanne, P.; Rodier, J.; Chavel, P.; Cambril, E.; Chen, Y. Imaging with blazed-binary diffractive elements. Journal of Optics A: Pure and Applied Optics 2002, 4, S119.

(7) Zhu, A. Y.; Kuznetsov, A. I.; Luk'yanchuk, B.; Engheta, N.; Genevet, P. Traditional and emerging materials for optical metasurfaces. Nanophotonics 2017, 6, 452-471.

(8) Genevet, P.; Capasso, F.; Aieta, F.; Khorasaninejad, M.; Devlin, R. Recent advances in planar optics: from plasmonic to dielectric metasurfaces. Optica 2017, 4, 139-152.

(9) Chen, H.-T.; Taylor, A. J.; Yu, N. A review of metasurfaces: physics and applications. Reports on progress in physics 2016, 79, 076401. 
(10) Xie, Y.-Y.; Ni, P.-N.; Wang, Q.-H.; Kan, Q.; Briere, G.; Chen, P.-P.; Zhao, Z.-Z.; Delga, A.; Ren, H.-R.; Chen, H.-D., et al. Metasurface-integrated vertical cavity surfaceemitting lasers for programmable directional lasing emissions. Nature nanotechnology 2020, 15, 125-130.

(11) Salandrino, A.; Engheta, N. Far-field subdiffraction optical microscopy using metamaterial crystals: Theory and simulations. Phys. Rev. B 2006, 74, 075103.

(12) Krasnok, A.; Tymchenko, M.; Alù, A. Nonlinear metasurfaces: a paradigm shift in nonlinear optics. Materials Today 2018, 21, 8 - 21.

(13) Bekenstein, R.; Pikovski, I.; Pichler, H.; Shahmoon, E.; Yelin, S.; Lukin, M. Quantum metasurfaces with atom arrays. Nature Physics 2020, 16, 676-681.

(14) Molesky, S.; Lin, Z.; Piggott, A. Y.; Jin, W.; Vuckovic, J.; Rodriguez, A. W. Outlook for inverse design in nanophotonics. arXiv preprint arXiv:1801.06715 2018,

(15) Yao, K.; Unni, R.; Zheng, Y. Intelligent nanophotonics: merging photonics and artificial intelligence at the nanoscale. Nanophotonics 2019, 8, 339-366.

(16) Elsawy, M. M. R.; Lanteri, S.; Duvigneau, R.; Brière, G.; Mohamed, M. S.; Genevet, P. Global optimization of metasurface designs using statistical learning methods. Scientific Reports 2019, 9 .

(17) Elsawy, M. M. R.; Lanteri, S.; Duvigneau, R.; Fan, J. A.; Genevet, P. Numerical Optimization Methods for Metasurfaces. Laser 63 Photonics Reviews 2020, 14, 1900445.

(18) Marler, R. T.; Arora, J. S. The weighted sum method for multi-objective optimization: new insights. Structural and multidisciplinary optimization 2010, 41, 853-862.

(19) Kim, I. Y.; De Weck, O. Adaptive weighted sum method for multiobjective optimization: a new method for Pareto front generation. Structural and multidisciplinary optimization 2006, 31, 105-116. 
(20) Naidu, K.; Mokhlis, H.; Bakar, A. A. Multiobjective optimization using weighted sum artificial bee colony algorithm for load frequency control. International Journal of Electrical Power \& Energy Systems 2014, 55, 657-667.

(21) Jafar-Zanjani, S.; Inampudi, S.; Mosallaei, H. Adaptive genetic algorithm for optical metasurfaces design. Scientific reports 2018, 8, 1-16.

(22) Tang, D.; Chen, L.; Liu, J. Visible achromatic super-oscillatory metasurfaces for subdiffraction focusing. Optics express 2019, 27, 12308-12316.

(23) Lalbakhsh, A.; Afzal, M. U.; Esselle, K. P. Multiobjective particle swarm optimization to design a time-delay equalizer metasurface for an electromagnetic band-gap resonator antenna. IEEE Antennas and Wireless Propagation Letters 2016, 16, 912-915.

(24) Allen, K. W.; Dykes, D. J.; Reid, D. R.; Lee, R. T. Multi-Objective Genetic Algorithm Optimization of Frequency Selective Metasurfaces to Engineer Ku-Passband Filter Responses. Progress In Electromagnetics Research 2020, 167, 19-30.

(25) Marler, R. T.; Arora, J. S. Survey of multi-objective optimization methods for engineering. Structural and multidisciplinary optimization 2004, 26, 369-395.

(26) Deb, K. Multi-objective optimization using evolutionary algorithms; John Wiley \& Sons, 2001; Vol. 16.

(27) Coello, C. A. C.; Lamont, G. B.; Van Veldhuizen, D. A., et al. Evolutionary algorithms for solving multi-objective problems; Springer, 2007; Vol. 5.

(28) Zhou, A.; Qu, B.-Y.; Li, H.; Zhao, S.-Z.; Suganthan, P. N.; Zhang, Q. Multiobjective evolutionary algorithms: A survey of the state of the art. Swarm and Evolutionary Computation 2011, 1, 32-49.

(29) Li, H.; Zhang, Q. Multiobjective optimization problems with complicated Pareto sets, 
MOEA/D and NSGA-II. IEEE transactions on evolutionary computation 2008, 13, $284-302$.

(30) Hadka, D.; Reed, P. Borg: An auto-adaptive many-objective evolutionary computing framework. Evolutionary computation 2013, 21, 231-259.

(31) Dhiman, G.; Singh, K. K.; Slowik, A.; Chang, V.; Yildiz, A. R.; Kaur, A.; Garg, M. EMoSOA: a new evolutionary multi-objective seagull optimization algorithm for global optimization. International Journal of Machine Learning and Cybernetics 2020, 1-26.

(32) Murata, T.; Ishibuchi, H. MOGA: multi-objective genetic algorithms. IEEE international conference on evolutionary computation. 1995; pp 289-294.

(33) Konak, A.; Coit, D. W.; Smith, A. E. Multi-objective optimization using genetic algorithms: A tutorial. Reliability Engineering \& System Safety 2006, 91, 992-1007.

(34) Deb, K.; Pratap, A.; Agarwal, S.; Meyarivan, T. A fast and elitist multiobjective genetic algorithm: NSGA-II. IEEE transactions on evolutionary computation 2002, 6, 182197.

(35) Igel, C.; Hansen, N.; Roth, S. Covariance matrix adaptation for multi-objective optimization. Evolutionary computation 2007, 15, 1-28.

(36) Coello, C. C.; Lechuga, M. S. MOPSO: A proposal for multiple objective particle swarm optimization. Proceedings of the 2002 Congress on Evolutionary Computation. CEC'02 (Cat. No. 02TH8600). 2002; pp 1051-1056.

(37) Mostaghim, S.; Teich, J. Strategies for finding good local guides in multi-objective particle swarm optimization (MOPSO). Proceedings of the 2003 IEEE Swarm Intelligence Symposium. SIS'03 (Cat. No. 03EX706). 2003; pp 26-33.

(38) Coello, C. A. C.; Pulido, G. T.; Lechuga, M. S. Handling multiple objectives with 
particle swarm optimization. IEEE Transactions on evolutionary computation 2004, 8, 256-279.

(39) Reyes-Sierra, M.; Coello, C. C., et al. Multi-objective particle swarm optimizers: A survey of the state-of-the-art. International journal of computational intelligence research 2006, 2, 287-308.

(40) Gagnon, D.; Dumont, J.; Dubé, L. J. Multiobjective optimization in integrated photonics design. Optics Letters 2013, 38, 2181-2184.

(41) Nagar, J.; Werner, D. H. A comparison of three uniquely different state of the art and two classical multiobjective optimization algorithms as applied to electromagnetics. IEEE Transactions on Antennas and Propagation 2017, 65, 1267-1280.

(42) Nagar, J.; Werner, D. H. Multiobjective Optimization for Electromagnetics and Optics: An Introduction and Tutorial Based on Real-World Applications. IEEE Antennas and Propagation Magazine 2018, 60, 58-71.

(43) Liu, Z.; Liu, X.; Xiao, Z.; Lu, C.; Wang, H.-Q.; Wu, Y.; Hu, X.; Liu, Y.-C.; Zhang, H.; Zhang, X. Integrated nanophotonic wavelength router based on an intelligent algorithm. Optica 2019, 6, 1367-1373.

(44) Allen, K. W.; Dykes, D. J.; Reid, D. R.; Bean, J. A.; Landgren, D. W.; Lee, R. T.; Denison, D. R. Metasurface engineering via evolutionary processes. 2017 IEEE National Aerospace and Electronics Conference (NAECON). 2017; pp 172-178.

(45) Wiecha, P. R.; Arbouet, A.; Girard, C.; Lecestre, A.; Larrieu, G.; Paillard, V. Evolutionary multi-objective optimization of colour pixels based on dielectric nanoantennas. Nature nanotechnology 2017, 12, 163.

(46) Wiecha, P. R.; Arbouet, A.; Girard, C.; Lecestre, A.; Larrieu, G.; Paillard, V. Multi- 
resonant silicon nanoantennas by evolutionary multi-objective optimization. Computational Optics II. 2018; p 1069402.

(47) Whiting, E. B.; Campbell, S. D.; Kang, L.; Werner, D. H. Meta-atom library generation via an efficient multi-objective shape optimization method. Opt. Express 2020, 28, 24229-24242.

(48) Campbell, S. D.; Zhu, D. Z.; Whiting, E. B.; Nagar, J.; Werner, D. H.; Werner, P. L. Advanced multi-objective and surrogate-assisted optimization of topologically diverse metasurface architectures. Metamaterials, Metadevices, and Metasystems 2018. 2018; p 107190U.

(49) So, S.; Badloe, T.; Noh, J.; Rho, J.; Bravo-Abad, J. Deep learning enabled inverse design in nanophotonics. Nanophotonics 2020,

(50) Yao, K.; Unni, R.; Zheng, Y. Intelligent nanophotonics: merging photonics and artificial intelligence at the nanoscale. Nanophotonics 2019, 8, 339-366.

(51) Wiecha, P. R.; Muskens, O. L. Deep learning meets nanophotonics: A generalized accurate predictor for near fields and far fields of arbitrary 3D nanostructures. Nano Letters 2019,

(52) Kiarashinejad, Y.; Zandehshahvar, M.; Abdollahramezani, S.; Hemmatyar, O.; Pourabolghasem, R.; Adibi, A. Knowledge Discovery in Nanophotonics Using Geometric Deep Learning. Advanced Intelligent Systems 2020, 2, 1900132.

(53) Kudyshev, Z. A.; Kildishev, A. V.; Shalaev, V. M.; Boltasseva, A. Machine-LearningAssisted Metasurface Design for High-Efficiency Thermal Emitter Optimization. arXiv preprint arXiv:1910.12741 2019,

(54) Kiarashinejad, Y.; Abdollahramezani, S.; Adibi, A. Deep learning approach based on 
dimensionality reduction for designing electromagnetic nanostructures. npj Computational Materials 2020, 6, 1-12.

(55) Campbell, S. D.; Sell, D.; Jenkins, R. P.; Whiting, E. B.; Fan, J. A.; Werner, D. H. Review of numerical optimization techniques for meta-device design. Optical Materials Express 2019, 9, 1842-1863.

(56) Inampudi, S.; Mosallaei, H. Neural network based design of metagratings. Applied Physics Letters 2018, 112, 241102.

(57) Liu, Z.; Zhu, D.; Rodrigues, S.; Lee, K.-T.; Cai, W. A Generative Model for the Inverse Design of Metasurfaces. Nano letters 2018,

(58) Jiang, J.; Fan, J. A. Global optimization of dielectric metasurfaces using a physicsdriven neural network. Nano letters 2019, 19, 5366-5372.

(59) Jiang, J.; Fan, J. A. Simulator-based training of generative neural networks for the inverse design of metasurfaces. Nanophotonics 2019, 1.

(60) Jiang, J.; Fan, J. A. Multiobjective and categorical global optimization of photonic structures based on ResNet generative neural networks. Nanophotonics 2020, 1.

(61) An, S.; Zheng, B.; Tang, H.; Shalaginov, M. Y.; Zhou, L.; Li, H.; Gu, T.; Hu, J.; Fowler, C.; Zhang, H. Generative multi-functional meta-atom and metasurface design networks. arXiv preprint arXiv:1908.04851 2019,

(62) Naseri, P.; Hum, S. V. A Generative Machine Learning-Based Approach for Inverse Design of Multilayer Metasurfaces. arXiv preprint arXiv:2008.02074 2020,

(63) Jones, D. Efficient global optimization of expensive black-box functions. Journal of Global Optimization 1998, 13.

(64) Jones, D. A taxonomy of global optimization methods based on response surfaces. Journal of Global Optimization 2001, 21, 345-383. 
(65) Forestiere, C.; He, Y.; Wang, R.; Kirby, R. M.; Dal Negro, L. Inverse design of metal nanoparticles' morphology. ACS Photonics 2015, 3, 68-78.

(66) Elsawy, M. M. R.; Lanteri, S.; Duvigneau, R.; Brière, G.; Mohamed, M. S.; Genevet, P. Global optimization of metasurface designs using statistical learning methods. Scientific Reports 2019, 9 .

(67) Gramacy, R. B. Surrogates: Gaussian Process Modeling, Design, and Optimization for the Applied Sciences; CRC Press, 2020.

(68) Shahriari, B.; Swersky, K.; Wang, Z.; Adams, R. P.; de Freitas, N. Taking the human out of the loop: A review of Bayesian optimization. Proceedings of the IEEE 2016, 104, 148-175.

(69) Emmerich, M. Single-and multi-objective evolutionary design optimization assisted by gaussian random field metamodels. Dissertation, LS11, FB Informatik, Universität Dortmund, Germany 2005,

(70) Palar, P. S.; Zuhal, L. R.; Chugh, T.; Rahat, A. On the impact of covariance functions in multi-objective Bayesian optimization for engineering design. AIAA Scitech 2020 Forum. 2020; p 1867.

(71) Emmerich, M. T.; Deutz, A. H.; Klinkenberg, J. W. Hypervolume-based expected improvement: Monotonicity properties and exact computation. Evolutionary Computation (CEC), 2011 IEEE Congress on. 2011; pp 2147-2154.

(72) Emmerich, M. T.; Giannakoglou, K. C.; Naujoks, B. Single-and multiobjective evolutionary optimization assisted by Gaussian random field metamodels. IEEE Transactions on Evolutionary Computation 2006, 10, 421-439.

(73) Binois, M.; Picheny, V. GPareto: An R Package for Gaussian-Process-Based MultiObjective Optimization and Analysis. Journal of Statistical Software 2019, 89, 1-30. 
(74) Binois, M.; Picheny, V. GPareto: Gaussian Processes for Pareto Front Estimation and Optimization. 2020; R package version 1.1.4.1.

(75) Lanteri, S.; Scheid, C.; Viquerat, J. Analysis of a generalized dispersive model coupled to a DGTD method with application to nanophotonics. SIAM J. Sci. Comp. 2017, 39, $831-859$.

(76) Viquerat, J. Simulation of electromagnetic waves propagation in nano-optics with a high-order discontinuous Galerkin time-domain method. Ph.D. thesis, University of Nice-Sophia Antipolis, 2015.

(77) Diogenes: A Discontinuous-Galerkin based software suite for nano-optics. https://diogenes.inria.fr/.

(78) Elsawy, M. M. R.; Hassan, K.; Boutami, S.; Lanteri, S. Bayesian optimization and rigorous modelling of a highly efficient 3D metamaterial mode converter. OSA Continuum 2020, 3, 1721-1729.

(79) Byrnes, S. J.; Lenef, A.; Aieta, F.; Capasso, F. Designing large, high-efficiency, highnumerical-aperture, transmissive meta-lenses for visible light. Optics express 2016, 24, 5110-5124.

(80) Wang, S.; Wu, P. C.; Su, V.-C.; Lai, Y.-C.; Chu, C. H.; Chen, J.-W.; Lu, S.-H.; Chen, J.; Xu, B.; Kuan, C.-H., et al. Broadband achromatic optical metasurface devices. Nature communications 2017, 8, 1-9.

(81) Wang, S.; Wu, P. C.; Su, V.-C.; Lai, Y.-C.; Chen, M.-K.; Kuo, H. Y.; Chen, B. H.; Chen, Y. H.; Huang, T.-T.; Wang, J.-H., et al. A broadband achromatic metalens in the visible. Nature nanotechnology 2018, 13, 227.

(82) Ndao, A.; Hsu, L.; Ha, J.; Park, J.-H.; Chang-Hasnain, C.; Kanté, B. Octave bandwidth photonic fishnet-achromatic-metalens. Nature communications 2020, 11, 1-6. 
(83) Balli, F.; Sultan, M.; Lami, S. K.; Hastings, J. T. A hybrid achromatic metalens. Nature communications 2020, 11, 1-8.

(84) Chung, H.; Miller, O. D. High-NA achromatic metalenses by inverse design. Optics Express 2020, 28, 6945-6965.

(85) Lin, Z.; Johnson, S. G. Overlapping domains for topology optimization of large-area metasurfaces. Optics express 2019, 27, 32445-32453.

(86) Lin, Z.; Liu, V.; Pestourie, R.; Johnson, S. G. Topology optimization of freeform largearea metasurfaces. Opt. Express 2019, 27, 15765-15775.

(87) Zhan, A.; Fryett, T. K.; Colburn, S.; Majumdar, A. Inverse design of optical elements based on arrays of dielectric spheres. Applied optics 2018, 57, 1437-1446.

(88) Backer, A. S. Computational inverse design for cascaded systems of metasurface optics. Optics express 2019, 27, 30308-30331.

(89) Mansouree, M.; McClung, A.; Samudrala, S.; Arbabi, A. Large-Scale Parametrized Metasurface Design Using Adjoint Optimization. ACS Photonics 2021, 8, 455-463.

(90) Zhang, D.; Qin, F.; Zhang, Q.; Liu, Z.; Wei, G.; Xiao, J. J. Segmented Bayesian optimization of meta-gratings for sub-wavelength light focusing. JOSA B 2020, 37, $181-187$.

(91) Pestourie, R.; Pérez-Arancibia, C.; Lin, Z.; Shin, W.; Capasso, F.; Johnson, S. G. Inverse design of large-area metasurfaces. Optics express 2018, 26, 33732-33747.

(92) Christiansen, R. E.; Sigmund, O. A tutorial for inverse design in photonics by topology optimization. arXiv preprint arXiv:2008.11816 2020,

(93) Yağcı, H. B.; Demir, H. V. "Meta-atomless" architecture based on an irregular continuous fabric of coupling-tuned identical nanopillars enables highly efficient and achromatic metasurfaces. Applied Physics Letters 2021, 118, 081105. 
(94) Liang, H.; Martins, A.; Borges, B.-H. V.; Zhou, J.; Martins, E. R.; Li, J.; Krauss, T. F. High performance metalenses: numerical aperture, aberrations, chromaticity, and trade-offs. Optica 2019, 6, 1461-1470.

(95) Presutti, F.; Monticone, F. Focusing on bandwidth: achromatic metalens limits. Optica 2020, 7, 624-631.

(96) Chen, W. T.; Zhu, A. Y.; Sisler, J.; Bharwani, Z.; Capasso, F. A broadband achromatic polarization-insensitive metalens consisting of anisotropic nanostructures. Nature communications 2019, 10, 1-7.

(97) Khadir, S.; Andrén, D.; Verre, R.; Song, Q.; Monneret, S.; Genevet, P.; Käll, M.; Baffou, G. Metasurface optical characterization using quadriwave lateral shearing interferometry. ACS photonics 2021, 8, 603-613. 